\title{
Multi-shuttle AS/RS Dimensions Optimization Using a Genetic Algorithm - Case of the Multi-aisle Configuration
}

wahiba FANDI ( $\sim$ fandi_wahiba@hotmail.com )

Universite Abou Bekr Belkaid Tlemcen Faculte de Technologie

\section{Sihem KOULOUGHLI}

Universite Abou Bekr Belkaid Tlemcen Faculte de Technologie

Latefa GHOMRI

Universite Abou Bekr Belkaid Tlemcen Faculte de Technologie

\section{Research Article}

Keywords: Multi-aisle AS/RS, Multi-shuttle S/R machine, Optimization , Multi-cycles , Optimal dimensions, Genetic algorithm

Posted Date: September 20th, 2021

DOI: https://doi.org/10.21203/rs.3.rs-903118/v1

License: (a) (1) This work is licensed under a Creative Commons Attribution 4.0 International License. Read Full License

Version of Record: A version of this preprint was published at The International Journal of Advanced Manufacturing Technology on February 11th, 2022. See the published version at https://doi.org/10.1007/s00170-022-08787-z. 


\title{
Multi-shuttle AS/RS dimensions optimization using a genetic algorithm - Case of the multi-aisle configuration
}

\author{
Wahiba Fandi* · Sihem Kouloughli · Latefa \\ Ghomri
}

Received: date / Accepted: date

\begin{abstract}
Over the past few years, automated storage and retrieval systems (AS/RSs) have been increasingly improving. It is worth mentioning that multi-shuttle storage/retrieval (S/R) machines were gradually introduced to the market some years ago. These machines, which possess a high speed of execution, are able to transport several pallets at a time during the same trip, as opposed to single-shuttle S/R machines which can carry only one pallet at a time. It should be noted that the installation of this type of system requires a significant financial investment, and therefore it is highly recommended that this system be well studied and designed prior to its installation. It is widely acknowledged that one of the most important objectives while designing an AS/RS is to achieve the shortest time for one single cycle. The present work aims at designing an AS/RS with optimal dimensions for the purpose of minimizing the time in a multi-cycle implementation. To do this, it was decided to consider a multi-aisle automated storage/ retrieval system (AS/RS) with a multi-shuttle S/R machine. In addition, a genetic algorithm (GA) was used for the optimization of the system.
\end{abstract}

Keywords Multi-aisle AS/RS · Multi-shuttle S/R machine · Optimization • Multi-cycles · Optimal dimensions · Genetic algorithm

\section{Introduction}

It is broadly admitted that using automated storage and retrieval systems (AS/RSs) in companies offers remarkable advantages. Indeed, this allows reducing storage costs, minimizing labor costs, using the storage space efficiently, and achieving a better traceability of products.

Wahiba Fandi*, Sihem Kouloughli · Latefa Ghomri Manufacturing Engineering Laboratory of Tlemcen Abou Bekr Belkaid University of Tlemcen, Algeria E-mail: wahiba.fandi@univ-tlemcen.dz

E-mail: kouloughli_sihem@yahoo.fr

E-mail: latefa.ghomri@univ-tlemcen.dz 
An automated storage/ retrieval system (AS/RS) consists of storage rack structures, storage and retrieval machines, conveyors and pickup/ delivery (P/D) stations. A storage/retrieval (S/R) machine is supposed to load and unload products.

It is worth indicating that many AS/RS configurations have been developed by considering the unit-load AS/RS which represents the generic system that can handle different types of products in terms of dimensions and volume. These configurations are different with regard to the arrangement of racks and the number of S/R machines included in the system. They are composed of a mini-load AS/RS, a carousel AS/RS, a mobile rack AS/RS, a flow rack AS/RS and a multi-aisle AS/RS.

The present study concentrates on the multi-aisle automated storage / retrieval system (AS/RS) which contains one single S/R machine in addition to a certain number of racks. Note it is important to mention that each pair of racks is separated by a service aisle. In addition, a common aisle, perpendicular to all racks, links all service aisles.

This type of system is highly advantageous as it necessitates the use of one single machine; in addition, it allows saving a lot of money as the installation costs of the automated storage/ retrieval system (AS/RS) represents about $40 \%$ [15] of the initial total investment. Moreover, the configuration with a large number of aisles allows handling and storing a large number of products.

Several researchers have attempted to improve the performance of the different types of the existing automated storage/ retrieval systems. In particular, they tried to minimize the cycle time of the S/R machine. According to the literature, this time parameter has in fact received much attention from researchers. It should be acknowledged that designing efficient automated storage/ retrieval systems is no easy task, as these systems need to be highly optimized.

In this context, several parameters, like the dimensions of the system, need to be optimized in order to guarantee the shortest cycle time for the multi-aisle automated storage/ retrieval system.

The rest of this paper is organized as follows. Section 2 presents the state of the art as well as some important scientific works carried out on the topic of automated storage/ retrieval systems. Next, the different notations and assumptions used in the article are reported in section 3 . Then, section 4 gives a description of a multi-aisle AS/RS along with their functioning principle. In section 5 , the first part presents the single cycle time model of the multi-aisle AS/RS; then, the second part gives the model describing the travel time between two bins of the multi-aisle $\mathrm{AS} / \mathrm{RS}$, in the case where the storage is done in the same aisle and in the case where it is done on different aisles; finally, the third and last part exposes the multi-cycle time model of the multi-aisle AS / RS. Then in section 6, an attempt is made to solve the problem of AS/RS dimensions optimization. Section 7 gives some general views about genetic algorithms. In section 8 , the selected genetic algorithm is applied for the purpose of identifying the optimal dimensions of the multi-aisle automated storage/ retrieval system under study. Then section 9 presents and discusses the results of the study, and finally, section 10 summarizes the conclusions drawn from this work. 


\section{State of the art}

In an automated storage/ retrieval system (AS/RS) that is provided with one single shuttle, the $\mathrm{S} / \mathrm{R}$ machine is designed in such a way that it can carry a maximum of one product at a time. It can implement single-cycle or double-cycle operations. In a single cycle, the machine can perform either the storage or the destocking, while in a double cycle, it does the stocking and then the destocking.

To the best of our knowledge, the majority of scientific works carried out so far on automated storage/ retrieval systems have mainly focused on AS/RS with one single shuttle only. Most researchers sought to design, model and optimize the cycle time of the machines they developed.

Among the pioneer works that focused on the modeling and optimization of automated storage/ retrieval systems is the one carried out by [4] who succeeded in developing analytical models for single and dual cycle times of a unit-load AS/RS. They then optimized the dimensions of their system. With regard to [13], they were deeply interested in multi-aisle AS/RS; they proposed a single cycle time analytical model for the system they designed. In this model, the authors tried to represent each rack as a continuous rectangle. In the global analytical model, the authors consider the mean cycle time for all racks. On the other hand, [16] proposed an analytical model for the cycle time of a multi-aisle AS/RS by taking into consideration the acceleration and deceleration parameters of the S/R machine. Similarly, [9] developed a mathematical model for the single cycle time of a multi-aisle AS/RS. In their study, the entire system was approximated by a continuous parallelepiped. Unlike the model proposed by Hwang and Ko, this model is completely continuous.

Unlike the model proposed by [13], this model is completely continuous. Moreover, [22] suggested a linear regression-based approach for the calculation of the cycle time using class-based storage for a unit-load automated storage/ retrieval system (AS/RS).

In more recent works, researchers started to focus on more compact systems. In this type of machines, a rack is supposed to have a capacity of more than one item, which makes the storage and retrieval operations more complex and more time-consuming.

In this framework, it is worth citing a number of research works that were interested in compact automated storage/ retrieval systems. For instance, [18] developed an analytical cycle time model for a free-fall-flow-rack AS/RS fitted with a gravitational conveyor under a dedicated storage assignment policy. With regard to [23] they proposed several compact AS/RS cycle time models by varying the positions of the input/output stations. In another work [9], the authors established an analytical model that aimed to determine the optimal cycle time for a compact AS/RS by adopting the class storage policy.

In the following section, a series of studies dealing with the optimization of the dimensions of various types of single-shuttle automated storage/ retrieval systems.

In this context, [15] sought to determine the optimal dimensions of a multiaisle AS/RS for a minimal single cycle time. In a later work, [14] identified the op- 
timal dimensions of a multi-aisle AS/RS for a dual cycle time when the storage and retrieval requests were in the same aisle. Afterwards, they kept the dimensions of one system unchanged, while those of the other two systems were optimized. Similarly, [2] used a Tabu search algorithm and the simulated annealing algorithm to minimize the cycle time of a compact AS/RS with a gravitational conveyor. On the other hand, [10] succeeded in identifying the optimal dimensions of an AS/RS with a U-shaped gravitational conveyor and a single S/R machine that is based on a counting technique. In [11] these same authors applied accurate heuristic approaches to optimize the design of an AS/RS with a bi-directional conveyor with two S/R machines; one for storage and the other one for retrieval.

Over the past few years, AS/RS have been widely investigated, and then multishuttle S/R machines started to be gradually introduced into the market. In the system under study, the S/R machine allows several storage and retrieval operations to be carried out during the same cycle. It is worth specifying that multishuttle AS/RS systems have received much less attention from researchers than single-shuttle AS/RS systems; this is mainly due to the fact that these systems were quite new. Below, a number of studies dealing with multi-shuttle AS/RS are cited.

For instance, [17] established some analytical models to estimate the flow rates of a double-shuttle system and a triple-shuttle system. As for [21] they proposed heuristic cycle time models for single-shuttle and multi-shuttle AS/RS systems. Regarding [16] they carried out simulations on multi-shuttle systems in order to assess the cycle time of an AS/RS with a mini load. In addition, [1] developed an analytical model for the travel time of a double-shuttle AS/RS.

Furthermore, [20] used a genetic algorithm (GA) to solve the sequencing problem in a triple-shuttle AS/RS with class-based storage. Similarly, [24] used the Tabu search algorithm to solve the location assignment problem in a multi-shuttle AS/RS. On the other hand, [7] attempted to solve the shift-based sequencing problem for a double- shuttle AS/RS. As for [19] they investigated the joint optimization of multishuttle AS/RS scheduling under shared storage in a fuzzy and dynamic environment. They found out that, in areas where the main objective is to reduce the cycle time, the shared storage policy is appropriate and the fuzzy set theory can be an effective tool for modeling the uncertainties that are encountered in real applications.

In the recent past, artificial intelligence has gained great interest in the field of industry. In general, the majority of researchers use metaheuristics to study automated storage/ retrieval systems. Some of these research works are cited below.

In this framework, [5] employed the multi-objective ant colony optimization (ACO) for the purpose of deciding on the distribution of items within the AS/RS, while handling several types of goods. They indeed indicated that the type of storage and load accumulation depends on different factors like the survey factor, product height, storage and space utilization, and shipping route. Similarly, [3] performed a multi-objective optimization for the design of a shuttle-based automated storage/retrieval system using the non-dominated sorting genetic algorithm II. With regard to [6] they carried out a particle swarm optimization (PSO) to solve the problem of assigning a double-shuttle AS/RS to a unit load. 
It is worth recalling that the present study intends to minimize the multi-cycle time of the Multi-Aisle Automated Storage and Retrieval Systems (MA-AS/RS) supplied with a multi-shuttle $\mathrm{S} / \mathrm{R}$ machine. The main purpose is to determine the optimal AS/RS dimensions that allow minimizing the multi-cycle time of the system. This can be achieved through the use of a genetic algorithm (GA).

\section{Notations and assumptions}

\subsection{Notations}

\subsubsection{The notations listed below are used throughout the entire paper:}

M: $\quad$ Number of racks in a multi-aisle AS/RS,

$\mathrm{M} / 2$ : $\quad$ Number of aisles in the AS/RS,

$\mathrm{N}_{\mathrm{L}}$ : $\quad$ Number of bins per row,

$\mathrm{N}_{\mathrm{H}}$ : Number of bins per column,

$\mathrm{N}$ : Total number of bins $\mathrm{N}=\mathrm{M} \times \mathrm{N}_{\mathrm{L}} \times \mathrm{N}_{\mathrm{H}}$

$t_{h}$ : Horizontal travel time from the first bin to the last one on the same row of the rack,

$t_{\nu}$ : Vertical travel time from the first bin to the last one on the same column of the rack,

$t_{p}: \quad$ Travel time from the first aisle to the last one,

$t_{p}^{\prime}: \quad$ Travel time from one aisle to the adjacent one,

$t_{h}^{\prime}$ : Horizontal travel time from one bin to its next neighboring bin,

$t_{\nu}^{\prime}: \quad$ Vertical travel time from one bin to its next neighboring bin,

E(SC): Average single cycle time,

E(DC): Average dual cycle time,

$\mathrm{E}(\mathrm{TB}): \quad$ Average travel time between two bins in the AS/RS,

$\mathrm{E}(\mathrm{MC}): \quad$ Average multi-cycle time,

n: $\quad$ Capacity of the S/R machine; i.e. the number of pallets that the S/R machine can carry at a time,

k: $\quad$ Number of operations per cycle; i.e. $\mathrm{k}$ can at most be equal to $2 \mathrm{n}$

\subsection{Assumptions}

- The AS/RS is composed of M racks, $M / 2$ aisles and $N$ bins, with $N=M \times N_{L} \times N_{H}$

- The S/R machine can move vertically and horizontally simultaneously; this is known as the Chebyshev displacement.

- The loading/unloading times of pallets as well as the acceleration /deceleration of the S/R machine are neglected

- The access probability to any bin in the system is the same, and the storage and retrieval methodology is random.

- The pickup/delivery (P/D) station is located in the lower left corner of the rack. 


\section{Description and operation of a multi-aisle AS/RS}

\subsection{Description}

A multi-aisle AS/RS is composed of a set of fixed racks, arranged in pairs in a parallel way, and separated by aisles. Each of these aisles, called a service aisle, gives access to two parallel racks. A common aisle, perpendicular to all racks, connects all the service aisles. A storage/retrieval (S/R) machine serves all racks.

The S/R machine moves, simultaneously, both vertically and horizontally (Chebyshev displacement) along all aisles of the system. It should be mentioned that the $\mathrm{S} / \mathrm{R}$ machine can move along two horizontal axes (along the depth on the service aisle and along the common aisle) and also through a vertical axis (columns).

It is appropriate to specify that the multi-aisle AS/RS with a single-shuttle S/R machine has a low flow. Note that a multi-shuttle machine can be used to increase that flow. It is also worth adding that using a multi-aisle AS/RS is significantly advantageous due to its reduced cost. In addition, using a single S/R machine, such as a multi-shuttle S/R machine, allows reducing the cost price of the system in comparison with other types of machines that use several automated storage/ retrieval systems.

Initially, the S/R machine is always located at the pickup/delivery (P/D) station. At the end of each cycle, which may involve several operations, the S/R machine returns back to the initial position.

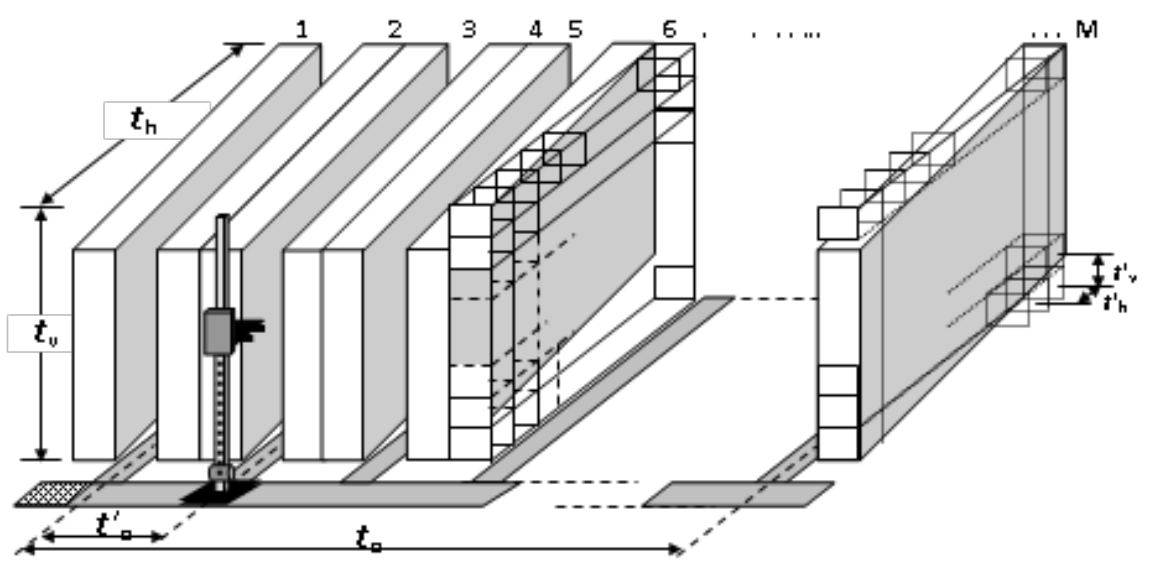

Fig. 1: Multi-aisle automated storage/retrieval system

4.2 Functioning of the storage/ retrieval (S/R) machine

Two types of S/R machines can be distinguished: the single-shuttle S/R machine and the multi-shuttle S/R machine. 
The single-shuttle S/R machine can carry a single pallet at a time, in which case it is said that the capacity of the machine is equal to one. Note that this kind of S/R machines can operate either in a single cycle mode or a dual cycle mode. In a single cycle, the $\mathrm{S} / \mathrm{R}$ machine performs a storage or retrieval operation and returns to the $\mathrm{P} / \mathrm{D}$ station, whereas, in a dual cycle, it performs a storage operation followed by a retrieval operation and then goes back to the P/D station.

With regard to the multi-shuttle $S / R$ machine, it can carry more than one pallet at a time. If for example this machine can carry a maximal number of $\mathrm{n}$ pallets, then it can operate single, dual, triple,..., (2n), cycles. In other words, if the S/R machine capacity is $n$, then it can maximally perform $n$ storage and $n$ retrieval operations in the same cycle. The time needed to complete this cycle is equivalent to the single cycle time plus $(2 n-1)$ multiplied by the time required to go from the storage bin to the retrieval bin (time needed to travel between two bins in the multi-aisle AS/RS).

On the other hand, a parameter $\mathrm{k}$ is introduced for the purpose of developing a general formula that allows assessing the multi-cycle time. This parameter represents the number of operations performed by an S/R machine of capacity $n$ during the same cycle; $\mathrm{k}$ is necessarily less or equal to $2 \mathrm{n}$.

The simplest form of S/R machines is the single-shuttle S/R machine $(n=1)$ where $\mathrm{k}$ may be equal either to one, if the machine executes one single cycle, or two if it executes a dual cycle.

Similarly, if the S/R machine can take two pallets at the same time $(n=2)$, then the parameter $\mathrm{k}$ can take the values 1,2, 3 and 4; and so on.

Likewise, the time required to execute a cycle during which the S/R machine carries out $\mathrm{k}$ storage and retrieval operations is equivalent to the single-cycle time plus $(\mathrm{k}-1)$ multiplied by the time required to go from the storage bin to the retrieval bin (travel time between two bins in the multi-aisle AS/RS). Consequently, the average multi-cycle time may be expressed as:

$$
\mathrm{E}(\mathrm{MC})=\mathrm{E}(\mathrm{SC})+(k-1) \times \mathrm{E}(\mathrm{TB})
$$

A quick literature review on the topic indicates that most researchers, who were interested in multi-shuttle automated storage/ retrieval systems, most of the time considered S/R machines with capacities of two or three, which means that these $\mathrm{S} / \mathrm{R}$ machines can perform a maximum of four (quadruple) cycles or six (sextuple) cycles.

Figure 2, presented below, depicts a cycle of the S/R machine that executes four operations. In this case, all four operations are supposed to take place in the same aisle. Obviously, this is not the general case, but it was deemed easy to represent on a figure. 


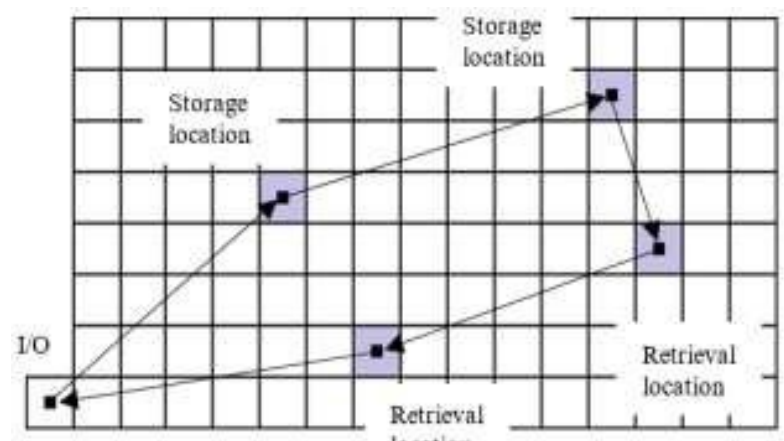

Fig. 2: Quadruple command cycle time

\section{Cycle time modeling for a multi-aisle AS/RS}

5.1 Analytical model for the expected single cycle time

As previously mentioned, in a single cycle, the S/R machine performs only one operation that can be either storage or retrieval operation.

In this context, it is worth citing the work of [9] who used a continuous approach to model the average single cycle time for a multi-aisle automated storage / retrieval system. This model is represented by equation (2) given below as:

$\mathrm{E}(\mathrm{SC})=\left\{\begin{array}{l}\frac{t_{p}+t_{h}}{2}+\frac{t_{v}^{3}}{24 \times t_{p} \times t_{h}} \text { if } t_{\nu} \leq \min \left(t_{p}, t_{h}\right) \\ \frac{t_{p}+t_{h}}{2}+\frac{t_{v}^{3}}{24 \times t_{p} \times t_{h}}-\frac{\left[t_{v}-\min \left(t_{p}, t_{h}\right)\right]^{4}}{24 \times t_{p} \times t_{h} \times t_{v}} \text { ifmin }\left(t_{p}, t_{h}\right)<t_{\nu} \leq \max \left(t_{p}, t_{h}\right) \\ \left.\frac{t_{p}+t_{h}}{2}+\frac{t_{v}^{3}}{24 \times t_{p} \times t_{h}}-\frac{\left[t_{v}-\min \left(t_{p}, t_{h}\right)\right]^{4}+\left[t_{v}-\max \left(t_{p}, t_{h}\right)\right]^{4}}{24 \times t_{p} \times t_{h} \times t_{v}} i f \max \left(t_{p}, t_{h}\right)<t_{v} \leq t_{p}+t_{h}\right) \\ \frac{t_{v}}{2}+\frac{2 \times\left(t_{p}+t_{h}\right)^{2}-\left(t_{p} \times t_{p}\right)}{12 \times t_{v}} i f t_{p}+t_{h}<t_{v}\end{array}\right.$

With regard to [14], they succeeded in demonstrating the continuity of the above equation (2) within the domain $\left(\mathrm{IR}^{*}+\right)^{3}$.

5.2 Analytical models for the expected travel time between two bins in the multi-aisle AS/RS

The expected travel time corresponds to the time period needed for the S/R machine to travel the distance separating two bins in the AS/RS. These bins may be either in the same aisle or in two different aisles. Consequently, the average travel time E (TB) between two bins in the AS/RS may be viewed as representing the analytical model for each of the two cases: 
- First case: The storage and retrieval operations are performed in the same aisle.

In this case, the model representing the time between two bins is similar to that of a unit-load AS/RS model which was established by [4] and expressed as:

$$
\mathrm{E}(\mathrm{TB})=\frac{\mathrm{T}}{3}+\frac{\left(\mathrm{T} b^{2}\right)}{6}-\frac{\left(\mathrm{T} b^{3}\right)}{30}
$$

Where $\mathrm{T}=\max \left(t_{\nu}, t_{h}\right)$ and $\mathrm{b}=\min \left(\frac{t_{v}}{\mathrm{~T}}, \frac{t_{h}}{\mathrm{~T}}\right)$

- Second case: The storage and retrieval operations are performed in different aisles.

In this case, the function $\mathrm{E}(\mathrm{TB})$ is written in the form of 18 restrictions, and each restriction corresponding to a specific domain of definition, as indicated in the appendix. As these restrictions are too large to be reported at this point, it was deemed wiser to write them in the appendix.

Note that, for the single cycle model, the E (TB) function was shown to be continuous on domain $\left(\mathrm{IR}^{*}+\right)^{3}$.

\subsection{Analytical models for the expected multi-cycle time}

A multi cycle consists of several storage and retrieval operations. In this case, the $\mathrm{S} / \mathrm{R}$ machine performs one single cycle and several additional displacements in order to execute a multi cycle.

The time needed to accomplish a multi cycle containing $\mathrm{k}$ operations is equal to one single cycle time plus $(\mathrm{k}-1)$ multiplied by the time required to go from the storage bin to the retrieval bin (travel time between two bins in the multiaisle AS/RS), as is expressed in Equation (1). Therefore, it may be deduced that the multi-cycle time function is continuous on domain $\left(\mathrm{IR}^{*}+\right)^{3}$.

Let's specify that the primary aim of this paper consists in optimizing the multi cycle time in order to determine the optimal dimensions of the multi-aisle AS/RS. The first step involves the formulation of the problem.

\section{Description of the research problem}

Designing an automated storage/ retrieval system (AS/RS) consists in determining its external appearance. This would involve a number of essential aspects such as the choice of AS/RS type, number of aisles, number of racks, number and type of S/R machines employed and dimensions of the system.

The present paper focuses primarily on determining the type of S/R machine as well as the optimal dimensions of the multi-aisle automated storage/ retrieval system to be selected. It was revealed that the installation of a multi-aisle AS/RS supplied with a multi-shuttle machine costs about one and a half the cost price of the installation of the same system with a single-shuttle machine [8] this represents a significant investment which is supposed to be recouped as soon as possible. This would certainly be possible only if the multi-shuttle system can considerably improve the throughput of the system in comparison with a single-shuttle 
system. Let's recall that one of the objectives of this paper is to assess the throughput performance of the multi-aisle AS/RS when the capacity of the S/R machine increases.

Furthermore, in order to identify the optimal dimensions of the multi-aisle AS/RS, it was deemed necessary to find an appropriate balance between the length of racks, their width and their height through cycle time minimization. It should be noted that the relationship between the time parameters $t_{p}, t_{h}$ and $t-v$ and AS/RS dimensions, i.e. length, width and depth, is quite evident since the horizontal and vertical speeds of the S/R machine are constant. Once again, this paper seeks to determine the optimal parameters $t_{p}, t_{h}$ and $t_{\nu}$ by minimizing the multicycle time. Bear in mind also that the complexity of the multi-cycle time equations makes the accurate analytical calculations quite difficult. For this reason, it was decided to apply the genetic algorithm (GA) to determine the optimal dimensions of the multi-aisle AS/RS.

\section{Genetic algorithm method}

Over the last years, several methods have been developed to solve optimization problems. These methods can be classified into two main categories: accurate methods and approximate methods.

Accurate methods are used to find the solutions of optimization problems with reasonable size. For example, some authors [14] used this type of method to solve the optimization problem of a single cycle time. They also solved the optimization problem of a dual cycle time when the storage and retrieval operations are performed in the same aisle.

Nevertheless, accurate methods can quickly reach their limits when the size of the problem is quite large. In the present case, the optimization problem of the dual cycle time becomes difficult to solve when using accurate methods due to the large number of operations to be performed.

Consequently, a new generation of approximate methods, called metaheuristics, has been developed to solve big problems, like our optimization problem where the equations are quite complex. Therefore, this paper seeks to find optimal solutions for this problem using genetic algorithms (GAs) which lately have become quite popular tools for solving similar problems [20].

It is widely known that genetic algorithms are stochastic optimization algorithms that are based on natural selection mechanisms and genetics. They were actually developed for the first time in 1975 [12]; he applied them to solve various combinatorial optimization problems.

The genetic algorithm (GA) can randomly manipulate an initial population of solutions (chromosomes) and then evaluate their relative performance (fitness). Afterwards, based on that performance, a new population of solutions is created using simple evolutionary operators such as selection, crossover and mutation in the form of a cycle that is repeated until a satisfactory solution is found. 
Genetic algorithms use the concept of populations of individuals to solve optimization problems because this principle allows achieving several optimal or near-optimal solutions.

\section{Optimization of multi-aisle AS/RS cycle time}

The purpose is to minimize the multi-cycle time in order to determine the optimal dimensions of the multi-aisle automated storage/ retrieval system by applying the genetic algorithm. This would involve defining the concepts of problem coding, initial population, crossover and mutation as well as determining the fitness function, the size of the population and the number of generations.

Consequently, in order to minimize the multi-cycle time, it was deemed necessary to consider that the following two constraints are related to our problem:

$$
t_{p}>0, t_{h}>0, t_{v}>0
$$

Here, $t_{p}, t_{v}$, and $t_{h}$ are time variables that are positive and different from zero.

$$
t_{p} \times t_{h} \times t_{\nu}=\text { constant }
$$

Here, the volume of the system is considered as constant. Therefore, if this volume is taken as constant and arbitrarily equal to 1 , then one may write: $\mathrm{t}_{v}=\frac{1}{\left(t_{p} \times t_{h}\right)}$

So, replacing $t_{\nu}$ by its values in equation (1) leads to a new function of two variables $t_{p}$ and $t_{h}$. This new function is called the fitness of the genetic algorithm.

\subsection{Problem coding}

The candidate solutions in a genetic algorithm (GA) are known as individuals or chromosomes characterized by a set of parameters (variables) known as genes. They can be binary- coded or real-coded. In the present case, a chromosome is the real pair $\left(t_{p}, t_{h}\right)$ that represents the temporal dimensions of the multi-aisle AS/RS. These dimensions are arranged in a specific sequence so that $t_{p}$ is in the first position and $t_{h}$ is in the second.

\subsection{Initial population}

An initial population of size 20 and a number of operations $k$, which starts with 1 , were first considered. In addition, in each chromosome there were genes $t_{p}$ and $t_{h}$ that were randomly selected within the interval [0-3].

Furthermore, the number of generations was set at 1000. Moreover, a minimal fitness function was maintained for each new generation. 


\subsection{Fitness function and individual selection}

The fitness function allows calculating the performance of each chromosome through the evaluation of the cycle time (equation (1)), for each chromosome. The chromosomes to reproduce are selected according to their fitness.

It is worth specifying that $50 \%$ of chromosomes with the smallest cycle times were chosen as a new population for the next generation. Note also that the selection of parent chromosomes was done according to the roulette wheel selection procedure. The probability of roulette selection is given by Equation (6) below.

$$
\mathrm{P}_{i}=\frac{f_{i}}{\sum_{i=0}^{s} f_{i}}
$$

Where $p_{i}$ is the probability for chromosome $\mathrm{i}, \mathrm{S}$ is the population size, and $f_{i}$ the fitness value of chromosome $i$.

Population diversity must be ensured over all generations in order to explore the research space as much as possible. This can be done by means of the crossover and mutation operators.

\subsection{Crossover}

A new population can be created from the selected parents using crossover operators. The parent chromosome that is expected to mate is selected in a haphazard way, and the number of mate chromosomes may be checked using the crossover rate (pc) parameter which, in this case, is defined as pc $=80 \%$.

In the literature, it was reported that several types of crossovers may be activated, i.e. one-point crossover, two-point crossover, three-point crossover, etc.... In our case, a one-point crossover was chosen between genes $t_{p}$ and $t_{h}$ of the selected parents.

The example illustrated in Figure 3 shows that the crossover will take place between two parent chromosomes $\left(i_{1}\right.$ and $\left.i_{2}\right)$.

Furthermore, one cut-point position was chosen at the middle point between chromosomes $i_{1}$ and $i_{2}$. The two terminal substrings of each chromosome were then exchanged, hence producing two children $i_{0} 1$ and $i_{0} 2$.

Afterwards, the resulting population undergoes a mutation process to create genetic diversity and avoid local solutions.

\subsection{Mutation}

The number of chromosomal mutations in a population is determined by the mutation parameter rate $(\mathrm{pm})$. The mutation process is carried out by randomly taking one of the two genes $\left(t_{p}\right.$ or $\left.t_{h}\right)$ and replacing its value by a new value.

The mutation parameter rate $(\mathrm{pm})$ was fixed at $10 \%$, which means that $10 \%$ of the total number of genes in the population is expected to undergo mutation. Then, the selected gene $\left(t_{p}\right.$ or $\left.t_{h}\right)$ is replaced by any randomly selected value within 
the range [0 - 3]. The example illustrated in Figure 4 shows that the second gene $\left(t_{h}\right)$ of the chromosome underwent a mutation and its value passed from 0.8891 to 0.8791 which was randomly taken from the interval [0 - 3].

The resulting new chromosomes are finally evaluated and integrated into the next generation which in turn undergoes the same process as the previous generation of chromosomes; they go through evaluation, selection, crossover and mutation. This then produces a new generation of chromosomes that will be used in the next iteration. This same process is repeated for all 1000 generations in order to obtain the best chromosome.

The best chromosomes, which represent the temporal dimensions of the multiaisle system for 1 to 50 operations, are summarized and discussed in the following section.

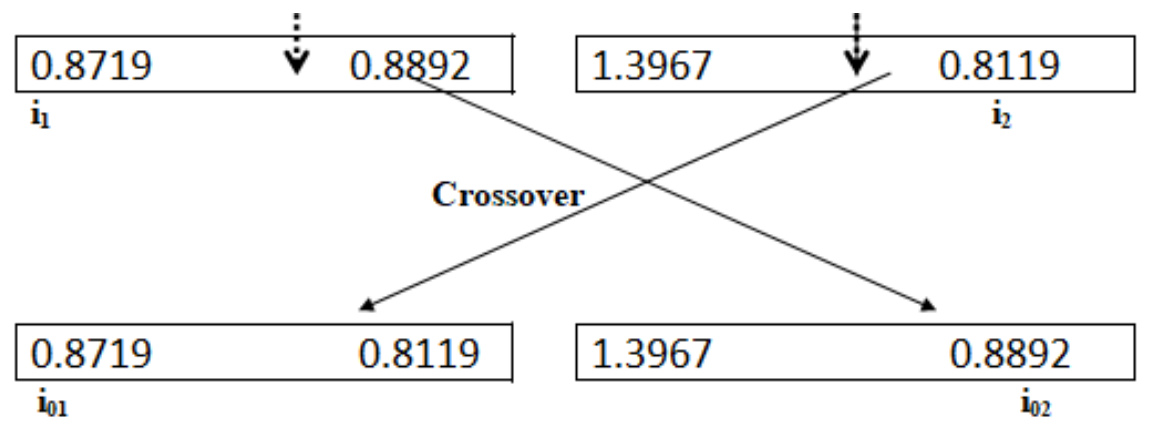

Fig. 3: Crossover operation

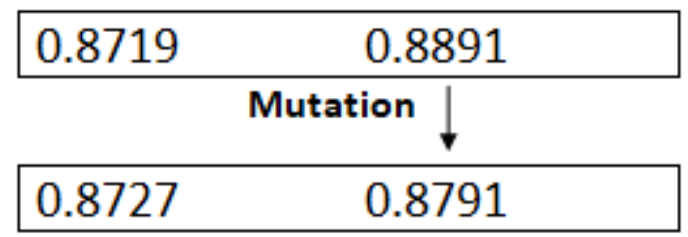

Fig. 4: Mutation process 


\section{Results and discussion}

The multi-shuttle S/R machine performs several operations in a cycle. The purpose is to minimize the cycle time in order to determine the optimal temporal dimensions of the multi-aisle automated storage/ retrieval system.

For this reason, it was decided to apply the genetic algorithm (GA) to minimize equation (1) while taking into account the constraints relating to the size of the system, which is assumed to be constant, and to the positivity of the temporal dimensions of the system. In addition, the value of $k$ was varied from 1 to 50 to see the evolution of dimensions with respect to the number of operations. In reality, this number is very large because in the majority of the work carried out, the capacity of the multi-shuttle S/R machines was limited to 2 or 3 for a quadruple or sextuple cycle time. However, it is believed that multi-shuttle S/R machines can perform well for a capacity greater than 3 for a multi cycle time.

Two different and separate cases are treated in this paper. In the first case, the multi-shuttle S/R machine performs storage and retrieval operations in the same aisle. In the second case, the multi-shuttle S/R machine performs the storage and retrieval operations in different aisles.

First case: the storage and retrieval operations are performed in the same aisle $\mathrm{K}=1$ : the single cycle time equation (2) is used

$\mathrm{k}>1$ : the multi-cycle time equation (1) is used while considering equation (3) for $\mathrm{E}$ (TB)

The results obtained, using the genetic algorithm (GA), are summarized in Table 1 .

Table 1 summarizes the optimal temporal dimensions $t_{p}, t_{h}$ and $t_{v}$ as well as their minimum cycle times obtained by applying the genetic algorithm in the case where the multi-shuttle S/R machine performs 1 to 50 storage and retrieval operations in the same aisle.

Note that, in the case where $\mathrm{k}=1$, the optimal dimensions $t_{p}, t_{h}$ and $t_{\nu}$ are respectively equal to $0.8710,0.8761$, and 1.3103 . However, for $\mathrm{k}=2$, these optimal dimensions are respectively equal to $0.9963,0.8334$, and 1.2042 .

The results obtained in the present work are in agreement with those found by [14] for a single cycle time and a dual cycle time.

Finally, the optimal dimensions of $t_{p}, t_{h}$ and $t_{v}$ are plotted against the number of operations k (Figure 5).

Figure 5 displays the evolution of the dimensions $t_{p}, t_{h}$ and $t_{v}$ as a function of the number of operations $\mathrm{k}$.

For $\mathrm{k} \in[1-50]$, it is observed that the value of $t_{p}$ increases continuously as the number of transactions augments.

For $\mathrm{k} \in[1-10]$, the values of $t_{\nu}$ and $t_{h}$ decrease as the number of transactions rises.

For $\mathrm{k} \in[10-50]$, the previous dimensions, continue to decrease. Note that the values of $t_{\nu}$ and $t_{h}$ are approximate.

On the other hand, it was deemed useful to determine the approximate functions of $t_{p}$ and $t_{h}$ with the least squares method, using the Least Squares approx- 
Table 1: Optimal dimensions of a multi-aisle AS/RS for the case where the storage and retrieval are performed in the same aisle

\begin{tabular}{|c|c|c|c|c|}
\hline k & $t_{p}$ & $t_{h}$ & $t_{v}$ & $\mathrm{E}(\mathrm{MC})$ \\
\hline 1 & 0.8710 & 0.8762 & 1.3103 & 1.9868 \\
\hline 2 & 0.9963 & 0.8334 & 1.2043 & 2.4876 \\
\hline 3 & 1.1146 & 0.8004 & 1.1210 & 2.9563 \\
\hline 4 & 1.2360 & 0.7743 & 1.0448 & 3.3985 \\
\hline 5 & 1.3407 & 0.7558 & 0.9869 & 3.8179 \\
\hline 6 & 1.4610 & 0.7342 & 0.9323 & 4.2177 \\
\hline 7 & 1.5785 & 0.7159 & 0.8849 & 4.6007 \\
\hline 8 & 1.6936 & 0.6978 & 0.8462 & 4.9691 \\
\hline 9 & 1.8076 & 0.6777 & 0.8164 & 5.3247 \\
\hline 10 & 1.9236 & 0.6614 & 0.7860 & 5.6691 \\
\hline 11 & 2.0214 & 0.6514 & 0.7594 & 6.0035 \\
\hline 12 & 2.1279 & 0.6402 & 0.7341 & 6.3289 \\
\hline 13 & 2.2501 & 0.6234 & 0.7129 & 6.6462 \\
\hline 14 & 2.3391 & 0.6123 & 0.6982 & 6.9561 \\
\hline 15 & 2.4218 & 0.6051 & 0.6824 & 7.2594 \\
\hline 16 & 2.5441 & 0.5917 & 0.6643 & 7.55640 \\
\hline 17 & 2.6259 & 0.5871 & 0.6487 & 7.84774 \\
\hline 18 & 2.7283 & 0.5761 & 0.6362 & 8.13368 \\
\hline 19 & 2.8218 & 0.5688 & 0.6230 & 8.4148 \\
\hline 20 & 2.9040 & 0.5625 & 0.6122 & 8.6913 \\
\hline 21 & 2.9852 & 0.5549 & 0.6037 & 8.9634 \\
\hline 22 & 3.0816 & 0.5452 & 0.5952 & 9.2314 \\
\hline 23 & 3.1722 & 0.5404 & 0.5833 & 9.4956 \\
\hline 24 & 3.2789 & 0.5310 & 0.5743 & 9.7562 \\
\hline 25 & 3.3406 & 0.5272 & 0.5678 & 10.0133 \\
\hline 26 & 3.4102 & 0.5215 & 0.5623 & 10.2673 \\
\hline 27 & 3.5247 & 0.5140 & 0.5520 & 10.5180 \\
\hline 28 & 3.6044 & 0.5082 & 0.5459 & 10.7658 \\
\hline 29 & 3.7046 & 0.5030 & 0.5366 & 11.0109 \\
\hline 30 & 3.7744 & 0.4997 & 0.5302 & 11.2532 \\
\hline 31 & 3.8310 & 0.4965 & 0.5258 & 11.4929 \\
\hline 32 & 3.9420 & 0.4890 & 0.5188 & 11.7302 \\
\hline 33 & 3.9977 & 0.4842 & 0.5167 & 11.9651 \\
\hline 34 & 4.0665 & 0.4831 & 0.5091 & 12.1976 \\
\hline 35 & 4.1583 & 0.4789 & 0.5021 & 12.4280 \\
\hline 36 & 4.2354 & 0.4745 & 0.4976 & 12.6563 \\
\hline 37 & 4.2881 & 0.4702 & 0.4959 & 12.8824 \\
\hline 38 & 4.3995 & 0.4642 & 0.4897 & 13.1068 \\
\hline 39 & 4.4651 & 0.4610 & 0.4858 & 13.3291 \\
\hline 40 & 4.4946 & 0.4612 & 0.4824 & 13.5497 \\
\hline 41 & 4.5908 & 0.4556 & 0.4781 & 13.7683 \\
\hline 42 & 4.6664 & 0.4513 & 0.4748 & 13.9853 \\
\hline 43 & 4.7413 & 0.4503 & 0.4684 & 14.2007 \\
\hline 44 & 4.7984 & 0.4465 & 0.4667 & 14.4143 \\
\hline 45 & 4.8949 & 0.4417 & 0.4625 & 14.6265 \\
\hline 46 & 4.9110 & 0.4420 & 0.4606 & 14.8373 \\
\hline 47 & 5.0107 & 0.4386 & 0.4550 & 15.0463 \\
\hline 48 & 5.0943 & 0.4335 & 0.4528 & 15.2539 \\
\hline 49 & 5.1645 & 0.4294 & 0.4509 & 15.4604 \\
\hline 50 & 5.1970 & 0.4311 & 0.4463 & 15.6652 \\
\hline
\end{tabular}


imation in Maple. This would make it possible to directly obtain the value of the optimal dimension for a multi cycle time.

The approximate function of $t_{p}$ as given by the least squares method is given below:

$$
t_{p}(k) \approx t_{p}=6 \times 10^{-6} k^{3}-0.0011 k^{2}+0.1274 k+0.7429
$$

The relative standard deviation (RSD) represents the relative deviation between the dimensions of $t_{p}$, as given by the genetic algorithm, and those given by the approximate function $t_{p}(\mathrm{k})$. The maximum relative deviation is equal to $0.9215 \%$ (Table 2). The values of $t_{p}$ and the approximate function $t_{p}(\mathrm{~h})$ are represented as a function of $k$ in Figure 6.

The approximate function of $t_{h}$, as given by the least squares method, is expressed as:

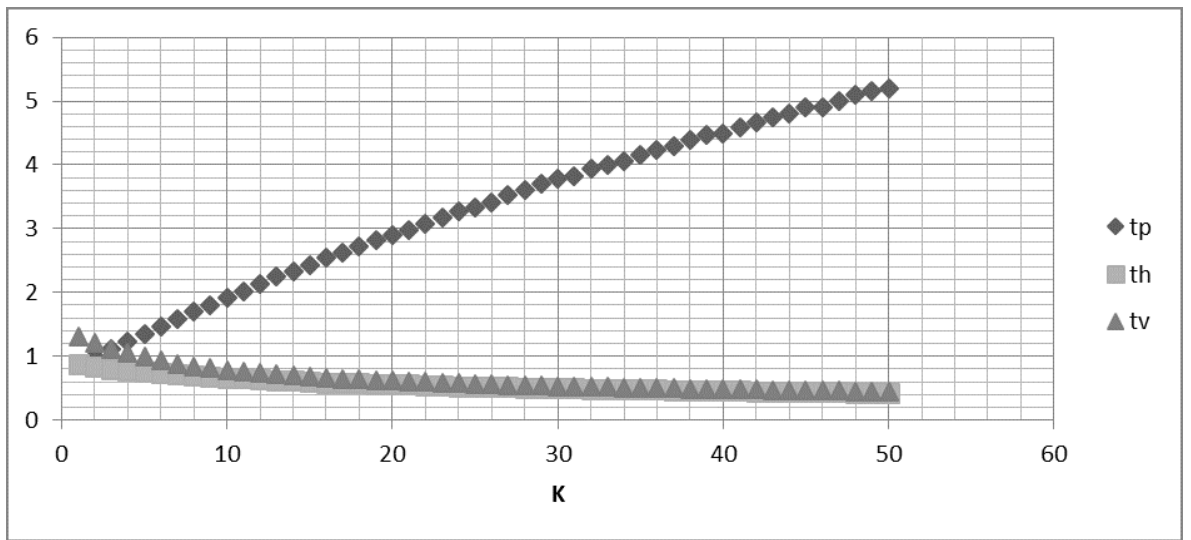

Fig. 5: Variation of the optimal dimensions $t_{p}, t_{h}$ and $t_{v}$ as a function of $\mathrm{k}$

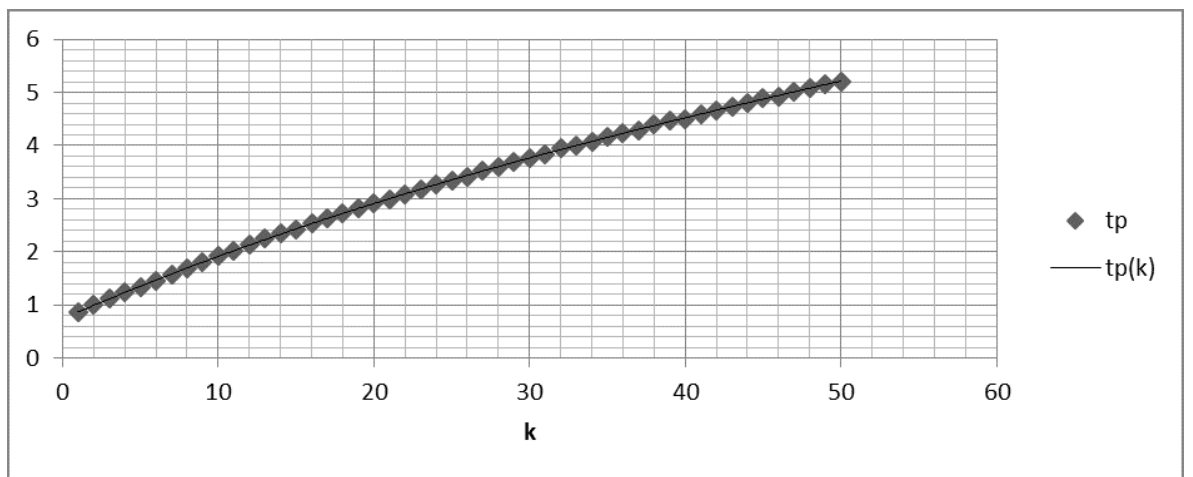

Fig. 6: Curve representing the optimal values of $t_{p}$ and the approximate function $t_{p}(\mathrm{k})$ 
Table 2: Relative standard deviation (RSD) between the optimal values of $t_{p}$ and the approximate function $t_{p}(\mathrm{k})$

\begin{tabular}{|c|c|c|c|}
\hline K & $t_{p}$ & $t_{p}(\mathrm{k})$ & RSD \\
\hline 1 & 0.8710 & 0.8690 & -0.2275 \\
\hline 2 & 0.9963 & 0.9931 & -0.3254 \\
\hline 3 & 1.1146 & 1.1151 & 0.0518 \\
\hline 4 & 1.2360 & 1.2350 & -0.0854 \\
\hline 5 & 1.3407 & 1.3530 & 0.9215 \\
\hline 6 & 1.4610 & 1.4690 & 0.5506 \\
\hline 7 & 1.5785 & 1.5831 & 0.2925 \\
\hline 8 & 1.6936 & 1.6953 & 0.1050 \\
\hline 9 & 1.8076 & 1.8057 & -0.1024 \\
\hline 10 & 1.9236 & 1.9143 & -0.4820 \\
\hline 11 & 2.0214 & 2.0212 & -0.0140 \\
\hline 12 & 2.1279 & 2.1263 & -0.0777 \\
\hline 13 & 2.2501 & 2.2297 & -0.9075 \\
\hline 14 & 2.3391 & 2.3314 & -0.3276 \\
\hline 15 & 2.4218 & 2.4316 & 0.4036 \\
\hline 16 & 2.5441 & 2.5302 & -0.5482 \\
\hline 17 & 2.6259 & 2.6272 & 0.0493 \\
\hline 18 & 2.7283 & 2.7227 & -0.2019 \\
\hline 19 & 2.8218 & 2.8168 & -0.1777 \\
\hline 20 & 2.9040 & 2.9095 & 0.1874 \\
\hline 21 & 2.9852 & 3.0008 & 0.5213 \\
\hline 22 & 3.0816 & 3.0907 & 0.2944 \\
\hline 23 & 3.1722 & 3.1792 & 0.2225 \\
\hline 24 & 3.2789 & 3.2666 & -0.3741 \\
\hline 25 & 3.3406 & 3.3527 & 0.3616 \\
\hline 26 & 3.4102 & 3.4376 & 0.8029 \\
\hline 27 & 3.5247 & 3.5214 & -0.0933 \\
\hline 28 & 3.6044 & 3.6040 & -0.0102 \\
\hline 29 & 3.7046 & 3.6855 & -0.5140 \\
\hline 30 & 3.7744 & 3.7660 & -0.2214 \\
\hline 31 & 3.8310 & 3.8455 & 0.3784 \\
\hline 32 & 3.9420 & 3.9240 & -0.4577 \\
\hline 33 & 3.9977 & 4.0016 & 0.0977 \\
\hline 34 & 4.0665 & 4.0782 & 0.2899 \\
\hline 35 & 4.1583 & 4.1541 & -0.1030 \\
\hline 36 & 4.2354 & 4.2290 & -0.1495 \\
\hline 37 & 4.2881 & 4.3033 & 0.3534 \\
\hline 38 & 4.3995 & 4.3767 & -0.5182 \\
\hline 39 & 4.4651 & 4.4495 & -0.3497 \\
\hline 40 & 4.4946 & 4.5215 & 0.6006 \\
\hline 41 & 4.5908 & 4.5930 & 0.0486 \\
\hline 42 & 4.6664 & 4.6638 & -0.0555 \\
\hline 43 & 4.7413 & 4.7341 & -0.1521 \\
\hline 44 & 4.7984 & 4.8039 & 0.1138 \\
\hline 45 & 4.8949 & 4.8732 & -0.4443 \\
\hline 46 & 4.9110 & 4.9420 & 0.6315 \\
\hline 47 & 5.0107 & 5.0105 & -0.0054 \\
\hline 48 & 5.0943 & 5.0785 & -0.3095 \\
\hline 49 & 5.1645 & 5.1463 & -0.3532 \\
\hline 50 & 5.1970 & 5.2137 & 0.3215 \\
\hline
\end{tabular}




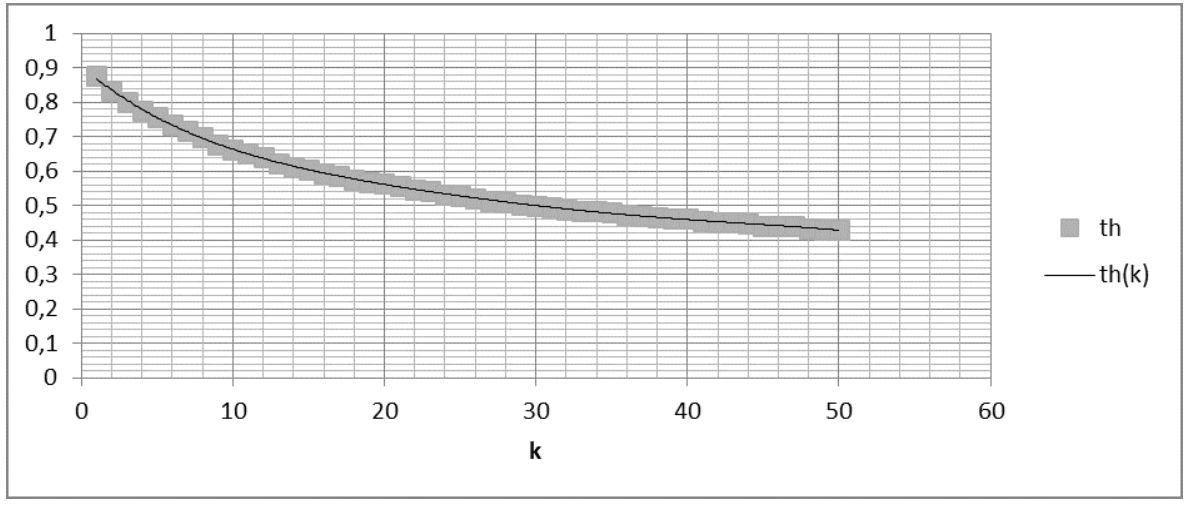

Fig. 7: Curve representing the optimal values of $t_{h}$ and the approximate function $t_{h}(\mathrm{k})$

$$
t_{h}(k) \approx t_{h}=-6 \times 10^{-9} k^{5}+9 \times 10^{-5} k^{4}-6 \times 10^{-5} k^{3}+0.0018 k^{2}-0.0377 k+0.9046
$$

The relative standard deviation (RSD) represents the relative difference between the horizontal dimensions, as given by the genetic algorithm, and those given by the approximate function $t_{h}(\mathrm{k})$. The maximum relative deviation is 0.8566 $\%$ (Table 3 ). The optimal values of $t_{h}$ and the approximate function $t_{h}(\mathrm{k})$ as a function of $k$ are shown in Figure 7.

Therefore, the form of the approximate function of $t_{v}$ can be deduced from Figure 6 in which one may see that the cloud of points corresponding to the optimal values of $t_{h}$ has the same shape as that of the points corresponding to the optimal values of $t_{v}$. It should also be noted that the optimal values of $t_{h}$ and $t_{v}$ are approximate.

Since $t_{h}(\mathrm{k})$ is a polynomial of degree 5 , so is the function $t_{\nu}(\mathrm{k})$.

The approximate function of $t_{\nu}$, as given by the least squares method, is:

$$
t_{\nu}(k) \approx t_{\nu}=-2 \times 10^{-8} k^{5}+4 \times 10^{-6} k^{4}-0.0002 k^{3}+0.0068 k^{2}-0.1111 k+1.4025
$$

As was previously done for the calculation of the relative deviation for $t_{p}(\mathrm{k})$ and $t_{h}(\mathrm{k})$, the maximum relative deviation between $t_{\nu}$ and $t_{v}(\mathrm{k})$ was found to be equal to $1.2921 \%$.

Second case: The storage and retrieval operations are performed in different aisles:

$\mathrm{h}=0$ : The single cycle time equation (2) is used

$\mathrm{h}>0$ : The multi-cycle time equation (1) is used while taking into account all 18 equations of $\mathrm{E}(\mathrm{TB})$ that are reported in the appendix.

The results obtained, using the genetic algorithm, are summarized in Table 4.

Table 4 summarizes the optimal temporal dimensions $t_{p}, t_{h}$ and $t_{v}$ and their minimum cycle times obtained by applying the genetic algorithm in the case where 
Table 3: Relative difference between the optimal values of $t_{h}$ and the approximate function $t_{h}(\mathrm{k})$

\begin{tabular}{|c|c|c|c|}
\hline $\mathrm{k}$ & $t_{h}$ & $t_{h}(\mathrm{k})$ & RSD \\
\hline 1 & 0.8762 & 0.8686 & -0.8567 \\
\hline 2 & 0.8334 & 0.8360 & 0.3131 \\
\hline 3 & 0.8004 & 0.8065 & 0.7592 \\
\hline 4 & 0.7743 & 0.7797 & 0.6919 \\
\hline 5 & 0.7558 & 0.7554 & -0.0575 \\
\hline 6 & 0.7342 & 0.7333 & -0.1230 \\
\hline 7 & 0.7159 & 0.7132 & -0.3685 \\
\hline 8 & 0.6978 & 0.6950 & -0.3986 \\
\hline 9 & 0.6777 & 0.6784 & 0.1099 \\
\hline 10 & 0.6614 & 0.6632 & 0.2771 \\
\hline 11 & 0.6514 & 0.6493 & -0.3278 \\
\hline 12 & 0.6402 & 0.6365 & -0.5743 \\
\hline 13 & 0.6234 & 0.6247 & 0.2045 \\
\hline 14 & 0.6123 & 0.6137 & 0.2321 \\
\hline 15 & 0.6051 & 0.6035 & -0.2548 \\
\hline 16 & 0.5917 & 0.5940 & 0.3842 \\
\hline 17 & 0.5871 & 0.5851 & -0.3445 \\
\hline 18 & 0.5761 & 0.5766 & 0.0899 \\
\hline 19 & 0.5688 & 0.5686 & -0.0263 \\
\hline 20 & 0.5625 & 0.5610 & -0.2609 \\
\hline 21 & 0.5549 & 0.5538 & -0.2008 \\
\hline 22 & 0.5452 & 0.5468 & 0.2938 \\
\hline 23 & 0.5404 & 0.5401 & -0.0541 \\
\hline 24 & 0.5310 & 0.5337 & 0.4987 \\
\hline 25 & 0.5272 & 0.5275 & 0.04862 \\
\hline 26 & 0.5215 & 0.5215 & -0.0037 \\
\hline 27 & 0.5140 & 0.5157 & 0.3292 \\
\hline 28 & 0.5082 & 0.5101 & 0.3668 \\
\hline 29 & 0.5030 & 0.5047 & 0.3384 \\
\hline 30 & 0.4997 & 0.4995 & -0.0362 \\
\hline 31 & 0.4965 & 0.4945 & -0.3918 \\
\hline 32 & 0.4890 & 0.4897 & 0.1506 \\
\hline 33 & 0.4842 & 0.4852 & 0.2096 \\
\hline 34 & 0.4831 & 0.4808 & -0.4722 \\
\hline 35 & 0.4789 & 0.4766 & -0.4767 \\
\hline 36 & 0.4745 & 0.4727 & -0.3871 \\
\hline 37 & 0.4702 & 0.4689 & -0.2868 \\
\hline 38 & 0.4642 & 0.4653 & 0.2470 \\
\hline 39 & 0.4610 & 0.4619 & 0.2034 \\
\hline 40 & 0.4612 & 0.4587 & -0.5378 \\
\hline 41 & 0.4556 & 0.4557 & 0.0209 \\
\hline 42 & 0.4513 & 0.4527 & 0.3069 \\
\hline 43 & 0.4503 & 0.4499 & -0.0982 \\
\hline 44 & 0.4465 & 0.4470 & 0.1160 \\
\hline 45 & 0.4417 & 0.4442 & 0.5659 \\
\hline 46 & 0.4420 & 0.4413 & -0.1597 \\
\hline 47 & 0.4386 & 0.4383 & -0.0654 \\
\hline 48 & 0.4335 & 0.4351 & 0.3568 \\
\hline 49 & 0.4294 & 0.4316 & 0.5046 \\
\hline 50 & 0.4311 & 0.4277 & -0.7996 \\
\hline
\end{tabular}


Table 4: Optimal dimensions of a multi-aisle AS / RS for the case where the storage and retrieval are performed in different aisles

\begin{tabular}{|c|c|c|c|c|}
\hline $\mathrm{k}$ & $t_{p}$ & $t_{h}$ & $t_{v}$ & EMC \\
\hline 1 & 0.8771 & 0.8771 & 1.2999 & 1.9868 \\
\hline 2 & 1.0315 & 0.6617 & 1.4652 & 3.0875 \\
\hline 3 & 1.1012 & 0.5903 & 1.5385 & 4.1168 \\
\hline 4 & 1.1236 & 0.5561 & 1.6005 & 5.1237 \\
\hline 5 & 1.1431 & 0.5361 & 1.6318 & 6.1204 \\
\hline 6 & 1.1689 & 0.5180 & 1.6515 & 7.1118 \\
\hline 7 & 1.1731 & 0.5072 & 1.6808 & 8.1001 \\
\hline 8 & 1.1841 & 0.5027 & 1.6799 & 9.0863 \\
\hline 9 & 1.1818 & 0.4967 & 1.7037 & 10.071 \\
\hline 10 & 1.1879 & 0.4923 & 1.7099 & 11.0547 \\
\hline 11 & 1.1918 & 0.4863 & 1.7254 & 12.0377 \\
\hline 12 & 1.1927 & 0.4861 & 1.7247 & 13.0201 \\
\hline 13 & 1.1967 & 0.4823 & 1.7327 & 14.002 \\
\hline 14 & 1.2054 & 0.4786 & 1.7335 & 14.9837 \\
\hline 15 & 1.1968 & 0.4777 & 1.749 & 15.9652 \\
\hline 16 & 1.2052 & 0.4732 & 1.7533 & 16.9462 \\
\hline 17 & 1.2007 & 0.4748 & 1.7541 & 17.9271 \\
\hline 18 & 1.2106 & 0.4703 & 1.7565 & 18.9077 \\
\hline 19 & 1.2027 & 0.4716 & 1.763 & 19.8885 \\
\hline 20 & 1.2118 & 0.4699 & 1.7559 & 21.8494 \\
\hline 21 & 1.2059 & 0.4687 & 1.7691 & 21.8494 \\
\hline 22 & 1.2146 & 0.4682 & 1.7587 & 22.8295 \\
\hline 23 & 1.2144 & 0.4641 & 1.7745 & 23.81 \\
\hline 24 & 1.2119 & 0.4673 & 1.7657 & 24.7898 \\
\hline 25 & 1.2169 & 0.4665 & 1.7615 & 25.7702 \\
\hline 26 & 1.2145 & 0.4649 & 1.771 & 26.7499 \\
\hline 27 & 1.2137 & 0.4634 & 1.775 & 27.7299 \\
\hline 28 & 1.2157 & 0.4625 & 1,775 & 28.7098 \\
\hline 29 & 1.2129 & 0.4624 & 1.7829 & 29.69 \\
\hline 30 & 1.2143 & 0.4601 & 1.7811 & 30.6697 \\
\hline 31 & 1.2197 & 0.4602 & 1.7821 & 31.6495 \\
\hline 32 & 1.2212 & 0.4631 & 1.7793 & 32.6292 \\
\hline 33 & 1.2142 & 0.4619 & 1.7784 & 33.6093 \\
\hline 34 & 1.2189 & 0.4579 & 1.7761 & 34.589 \\
\hline 35 & 1.2252 & 0.46 & 1.7824 & 35.5687 \\
\hline 36 & 1.2212 & 0.4584 & 1.78 & 36.5483 \\
\hline 37 & 1.2282 & 0.4594 & 1.7759 & 37.5286 \\
\hline 38 & 1.2213 & 0.4576 & 1.7822 & 38.5077 \\
\hline 39 & 1.2188 & 0.4595 & 1.793 & 39.4878 \\
\hline 40 & 1.2209 & 0.4598 & 1.7827 & 40.4671 \\
\hline 41 & 1.2198 & 0.4581 & 1.783 & 41.4468 \\
\hline 42 & 1.2159 & 0.458 & 1.7952 & 42.4269 \\
\hline 43 & 1.227 & 0.4561 & 1.7796 & 43.4065 \\
\hline 44 & 1.2202 & 0.4549 & 1.7967 & 44.3861 \\
\hline 45 & 1.2245 & 0.4569 & 1.7952 & 45.3656 \\
\hline 46 & 1.2278 & 0.4567 & 1.7828 & 46.3452 \\
\hline 47 & 1.2229 & 0.4573 & 1.7906 & 47.3242 \\
\hline 48 & 1.2221 & 0.4552 & 1.7894 & 48.3038 \\
\hline 49 & 1.2199 & 0.4585 & 1.8008 & 50.2634 \\
\hline 50 & 1.2178 & 0.4582 & 1.791 & 51.2429 \\
\hline
\end{tabular}




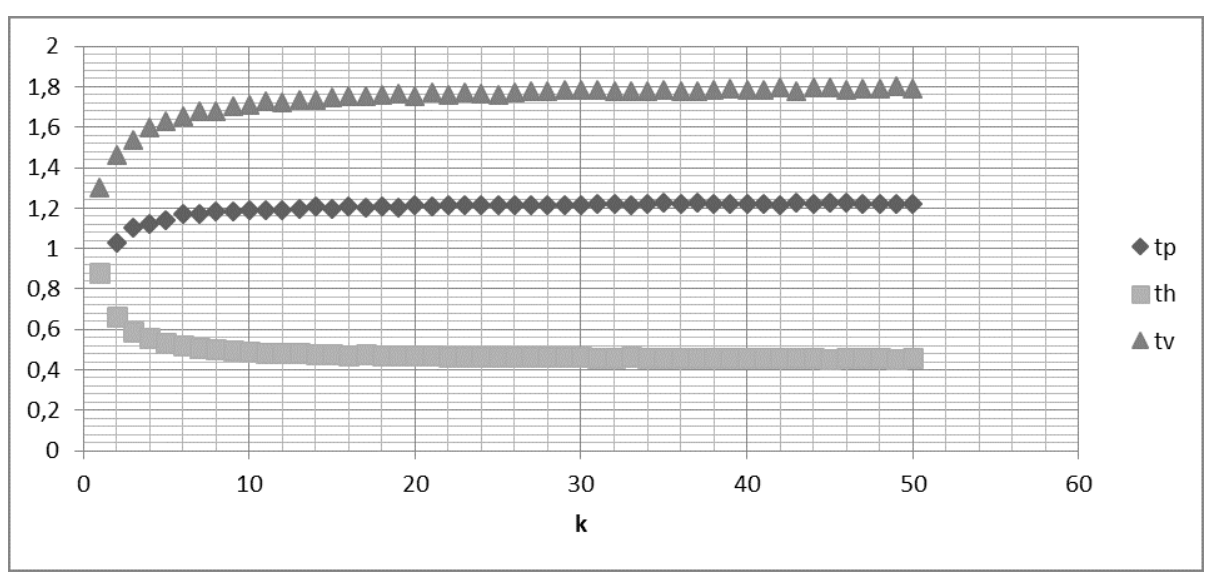

Fig. 8: Graph representing the optimal dimensions of $t_{p}, t_{h}$ and $t_{v}$ as a function of $\mathrm{k}$

the multi-shuttle S/R machine performs between 1 and 50 storage and retrieval operations in different aisles.

Table 4 shows that after $\mathrm{k}=16$ operations, the optimal dimensions are almost constant. Note that $t_{h} €[0.4585-0.4699], t_{p} €[1.2059-1.2178]$ and $t_{v} €[1.7559-$ 1.7910].

The graphical representation of the optimal dimensions of $t_{p}, t_{h}$ and $t_{\nu}$ as a function of the number of operations $\mathrm{k}$ is illustrated in Figure 8.

Figure 8 shows the evolution of the dimensions $t_{p}, t_{h}$ and $t_{v}$ as a function of $\mathrm{k}$.

One may clearly see that, for $\mathrm{k} €[1-16]$, the dimensions of $t_{p}$ and $t_{v}$ increase while those of $t_{h}$ decrease as the number of operations increases.

For $\mathrm{k} €[16-50]$, the three dimensions remain almost unchanged $\left(t_{h} €[0.4585-\right.$ $0.4699], t_{p} €[1.2059-1.2178]$ and $\left.t_{v} €[1.7559-1.7910]\right)$.

In addition, the approximate functions of $t_{p}$ and $t_{h}$ are determined with the least squares method, using the LeastSquares command in Maple. This makes it possible to directly obtain the values of the optimum dimensions for a multi cycle time.

The approximate function of $t_{p}$ as given by the least squares method, is then expressed as:

$$
t_{p}(k) \approx t_{p}=1.2187 \times\left(1-e^{-3134 k^{0.4708}}\right)
$$

The relative standard deviation (RSD) represents the relative deviation between the dimensions of $t_{p}$, as given by the genetic algorithm, and those given by the approximate function $t_{p}(\mathrm{k})$. The maximum relative deviation is equal to $1.5945 \%$ (Table 5)

The values of $t_{p}$ and the approximate function $t_{p}(\mathrm{k})$ as a function of $\mathrm{k}$ are represented in Figure 9.

With regard to the approximate function of $t_{h}$, it is given by the least squares method as: 
Table 5: Relative standard deviation (RSD) between the optimal values of $t_{p}$ and the approximate function $t_{p}(\mathrm{k})$

\begin{tabular}{llll}
\hline $\mathrm{k}$ & $t_{p}$ & $t_{p}(\mathrm{k})$ & $\mathrm{RSD}$ \\
\hline 1 & 0.8771 & 0.891 & 1.5945 \\
2 & 1.0315 & 1.0214 & -0.9782 \\
3 & 1.1012 & 1.0842 & -1.5444 \\
4 & 1.1236 & 1.121 & -0.2292 \\
5 & 1.1431 & 1.1448 & 0.1524 \\
6 & 1.1689 & 1.1613 & -0.65 \\
7 & 1.1731 & 1.1731 & -0.0048 \\
8 & 1.1841 & 1.1818 & -0.1891 \\
9 & 1.1818 & 1.1885 & 0.5687 \\
10 & 1.1879 & 1.1937 & 0.4878 \\
11 & 1.1918 & 1.1978 & 0.5041 \\
12 & 1.1927 & 1.2011 & 0.7006 \\
13 & 1.1967 & 1.2037 & 0.5871 \\
14 & 1.2054 & 1.2059 & 0.0384 \\
15 & 1.1968 & 1.2077 & 0.9084 \\
16 & 1.2052 & 1.2092 & 0.3317 \\
17 & 1.2007 & 1.2104 & 0.8117 \\
18 & 1.2106 & 1.2115 & 0.0768 \\
19 & 1.2027 & 1.2124 & 0.802 \\
20 & 1.2118 & 1.2132 & 0.1084 \\
21 & 1.2059 & 1.2138 & 0.6581 \\
22 & 1.2146 & 1.2144 & -0.0171 \\
23 & 1.2144 & 1.2149 & 0.0398 \\
24 & 1.2119 & 1.2153 & 0.2808 \\
25 & 1.2169 & 1.2157 & -0.1021 \\
26 & 1.2145 & 1.216 & 0.1224 \\
27 & 1.2137 & 1.2163 & 0.21 \\
28 & 1.2157 & 1.2165 & 0.0653 \\
29 & 1.2129 & 1.2168 & 0.3212 \\
30 & 1.2143 & 1.2169 & 0.219 \\
31 & 1.2197 & 1.2171 & -0.2082 \\
32 & 1.2212 & 1.2173 & -0.3218 \\
33 & 1.2142 & 1.2174 & 0.2637 \\
34 & 1.2189 & 1.2175 & -0.1156 \\
35 & 1.2252 & 1.2176 & -0.6206 \\
36 & 1.2212 & 1.2177 & -0.2868 \\
37 & 1.2282 & 1.2178 & -0.8481 \\
38 & 1.2213 & 1.2179 & -0.2752 \\
39 & 1.2188 & 1.218 & -0.0671 \\
40 & 1.2209 & 1.218 & -0.2342 \\
41 & 1.2198 & 1.2181 & -0.14 \\
42 & 1.2159 & 1.2182 & 0.183 \\
43 & 1.227 & 1.2182 & -0.7127 \\
44 & 1.2202 & 1.2183 & -0.1577 \\
45 & 1.2245 & 1.2183 & -0.5101 \\
46 & 1.2278 & 1.2183 & -0.7679 \\
47 & 1.2229 & 1.2184 & -0.3727 \\
48 & 1.2221 & 1.2184 & -0.3065 \\
50 & 1.2199 & 1.2184 & -0.124 \\
& 1.2178 & 1.2184 & 0.0538 \\
\hline & & & \\
& \\
& & \\
19 &
\end{tabular}




$$
t_{h}(k) \approx t_{h}=0.4494+e^{\left(-13.3255 k^{0.7229}+0.0071 k+12.4645\right)}
$$

It is worth noting that the relative standard deviation (RSD) represents the relative difference between the optimal values of $t_{h}$, as given by the genetic algorithm, and those given by the function $t_{h}(\mathrm{k})$. The maximum relative deviation is equal to $0.6053 \%$ (Table 6 ).

The values of $t_{h}$ and the approximate function $t_{h}(\mathrm{k})$ as a function of $\mathrm{k}$ are shown in Figure 10.

The form of the approximate function of $t_{v}$ can therefore be deduced from Figure 8 which indicates that the optimal values of $t_{p}$ are similar to the optimal values of $t_{\nu}$.

Since $t_{p}(\mathrm{k})$ is an increasing exponential function, so is $t_{v}(\mathrm{k})$.

Moreover, the approximate function of $t_{v}$, as given by the least squares method, is expressed as:

$$
t_{\nu}(k) \approx t_{\nu}=1.7960\left(1-e^{\left(-1.2960 k^{(0.3739)}\right)}\right)
$$

As the relative deviations have already been calculated for $t_{p}(\mathrm{k})$ and $t_{h}(\mathrm{k})$, then the maximum relative deviation between $t_{v}$ and $t_{v}(\mathrm{k})$ is equal to $0.6455 \%$.

The solutions found are the optimal dimensions that we propose when choosing the dimensions for the design of a multi-aisle AS/RS system with a multi-shuttle $\mathrm{S} / \mathrm{R}$ machine. They are summarized in Table 1 for the case where the storage and retrieval operations are performed in the same aisle and in Table 4 for the case where storage and retrieval are done in different aisles. It is worth recalling that the optimal dimensions were determined through minimization of the multi-cycle time and applying the genetic algorithm and also by considering that the number of bins in the system is constant (equation 5).

For a direct evaluation of the optimal dimensions of the system, it was decided to determine the functions that relate the optimal solutions $\left(t_{p}, t_{h}\right.$, and $\left.t_{v}\right)$ to the

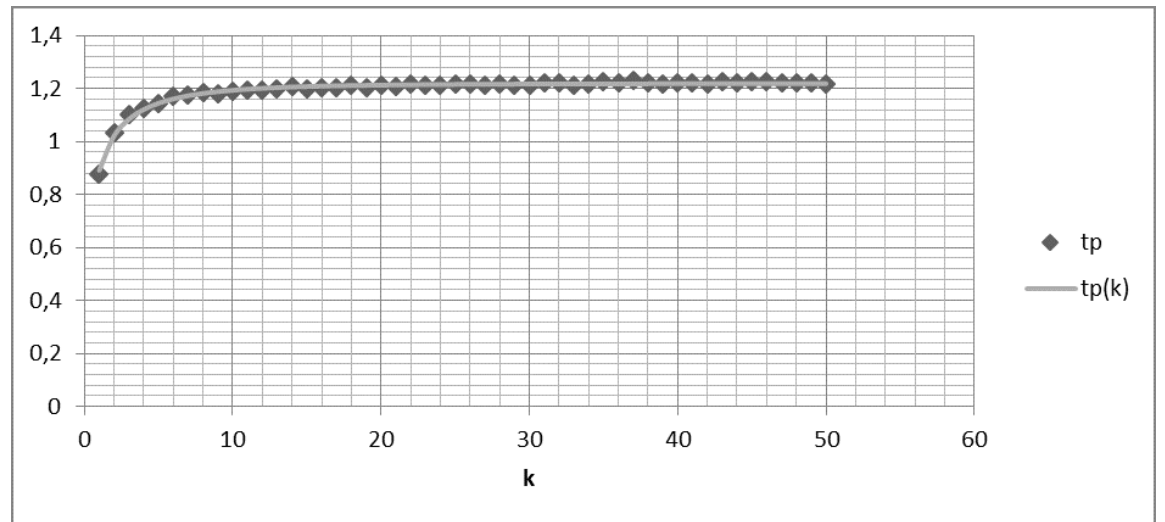

Fig. 9: Curve representing the optimal values of $t_{p}$ and the approximate function $t_{p}(\mathrm{k})$ 
Table 6: Relative standard deviation (RSD) between the optimal values of $t_{h}$ and the approximate function $t_{h}(\mathrm{k})$

\begin{tabular}{|c|c|c|c|}
\hline $\mathrm{k}$ & $t_{h}$ & $t_{h}(\mathrm{k})$ & RSD \\
\hline 1 & 0.8771 & 0.8752 & -0.2174 \\
\hline 2 & 0.6617 & 0.6657 & 0.6053 \\
\hline 3 & 0.5903 & 0.593 & 0.4693 \\
\hline 4 & 0.5561 & 0.5562 & 0.0312 \\
\hline 5 & 0.5361 & 0.5341 & -0.3722 \\
\hline 6 & 0.5180 & 0.5194 & 0.2575 \\
\hline 7 & 0.5072 & 0.5089 & 0.3367 \\
\hline 8 & 0.5027 & 0.501 & -0.3419 \\
\hline 9 & 0.4967 & 0.4949 & -0.346 \\
\hline 10 & 0.4923 & 0.4901 & -0.4447 \\
\hline 11 & 0.4863 & 0.4862 & -0.0232 \\
\hline 12 & 0.4861 & 0.4829 & -0.6553 \\
\hline 13 & 0.4823 & 0.4802 & -0.4315 \\
\hline 14 & 0.4786 & 0.4779 & -0.1473 \\
\hline 15 & 0.4777 & 0.4759 & -0.3956 \\
\hline 16 & 0.4732 & 0.4741 & 0.1803 \\
\hline 17 & 0.4748 & 0.4726 & -0.4699 \\
\hline 18 & 0.4703 & 0.4712 & 0.198 \\
\hline 19 & 0.4716 & 0.47 & -0.3369 \\
\hline 20 & 0.4699 & 0.4689 & -0.2168 \\
\hline 21 & 0.4687 & 0.4679 & -0.1701 \\
\hline 22 & 0.4682 & 0.4671 & -0.2314 \\
\hline 23 & 0.4641 & 0.4663 & 0.4777 \\
\hline 24 & 0.4673 & 0.4655 & -0.3793 \\
\hline 25 & 0.4665 & 0.4649 & -0.3482 \\
\hline 26 & 0.4649 & 0.4643 & -0.1409 \\
\hline 27 & 0.4634 & 0.4637 & -0.1 \\
\hline 28 & 0.4625 & 0.4632 & -0.0502 \\
\hline 29 & 0.4624 & 0.4627 & 0.051 \\
\hline 30 & 0.4601 & 0.4622 & -0.0268 \\
\hline 31 & 0.4602 & 0.4618 & 0.3826 \\
\hline 32 & 0.4631 & 0.4614 & 0.2646 \\
\hline 33 & 0.4619 & 0.4611 & -0.4397 \\
\hline 34 & 0.4579 & 0.4607 & -0.2546 \\
\hline 35 & 0.46 & 0.4604 & 0.5489 \\
\hline 36 & 0.4584 & 0.4601 & 0.0171 \\
\hline 37 & 0.4594 & 0.4598 & 0.3015 \\
\hline 38 & 0.4576 & 0.4596 & 0.0267 \\
\hline 39 & 0.4595 & 0.4593 & 0.3744 \\
\hline 40 & 0.4598 & 0.4591 & -0.0845 \\
\hline 41 & 0.4581 & 0.4588 & -0.2055 \\
\hline 42 & 0.458 & 0.4586 & 0.1103 \\
\hline 43 & 0.4561 & 0.4584 & 0.0972 \\
\hline 44 & 0.4549 & 0.4582 & 0.4583 \\
\hline 45 & 0.4569 & 0.458 & 0.6886 \\
\hline 46 & 0.4567 & 0.4579 & 0.2203 \\
\hline 47 & 0.4573 & 0.4577 & 0.2211 \\
\hline 48 & 0.4552 & 0.4575 & 0.0578 \\
\hline 49 & 0.4585 & 0.4574 & 0.4773 \\
\hline 50 & 0.4582 & 0.4572 & -0.2769 \\
\hline
\end{tabular}




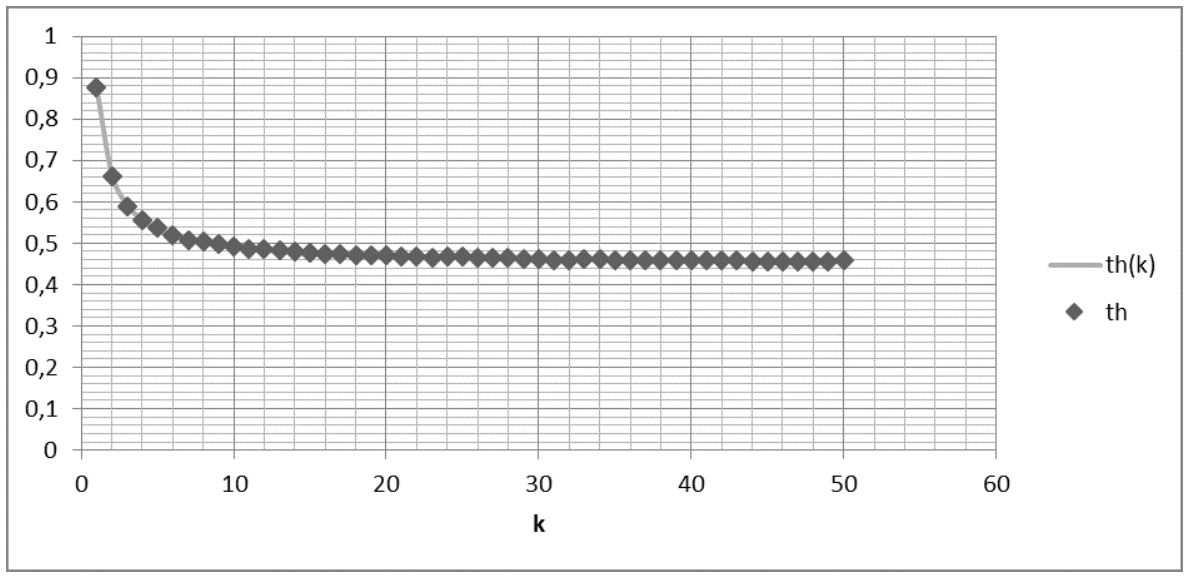

Fig. 10: Curve representing the optimal values of $t_{h}$ as well as the approximate function $t_{h}(\mathrm{k})$

number of operations $\mathrm{k}$, using the least squares method. A summary diagram of the results is presented in Figure 11. 


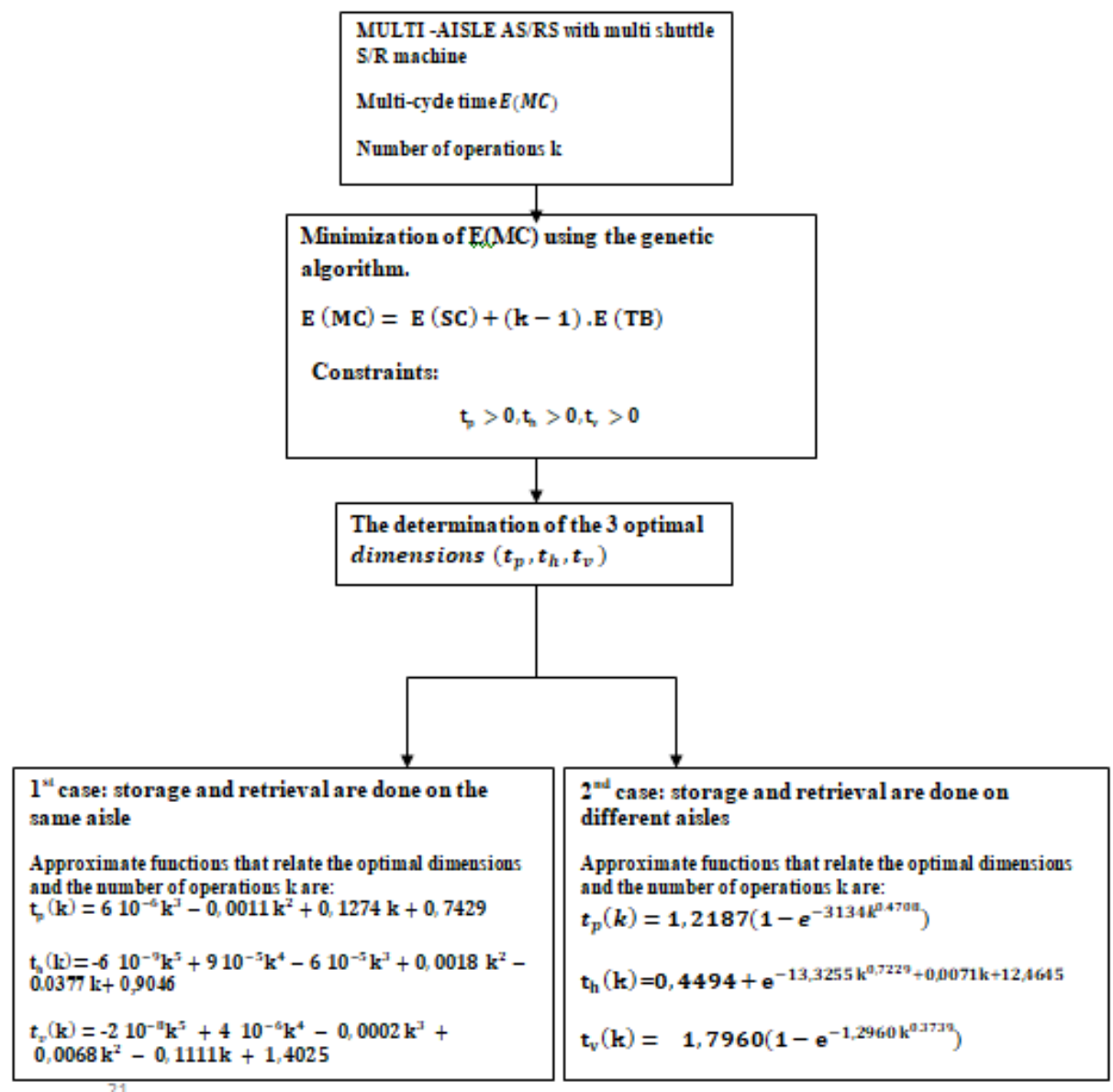

Fig. 11: Summary of results

\section{Conclusion}

The present article focused on automated storage and retrieval systems (AS/RSs), and particularly, on the multi-aisle AS/RS system with a multi-shuttle S/R machine.

This type of system is provided with a single S/R machine that can perform several storage and retrieval operations in the same cycle. This machine can circulate simultaneously in the horizontal and vertical directions to store or retrieve products in the system. It is worth recalling that the present work aims to find the optimal dimensions of a multi-aisle automated storage/retrieval system for the case where the storage and retrieval operations are done in the same aisle, and also for the case where these operations are performed in different aisles. 
The first step consisted in minimizing the multi cycle time E (MC) which was found equal to the single cycle time plus $(\mathrm{k}-1)$ multiplied by the time required to go from the storage bin to the retrieval bin (time between two bins in the multiaisle AS/RS).

This could be achieved by applying the genetic algorithm and considering that the number of bins in the system is constant. For this, it was decided to set a number of operations between 1 and 50 in order to see the evolution of dimensions according to the number of operations performed.

The results found are summarized in Table 1 for the case where the storage and retrieval operations are done in the same aisle, while Table 4 gives the results for the case where these operations are performed in different aisles. The data collected may be used when building the premises to house the multi-aisle automated storage/ retrieval system and therefore avoid constraints on the size of the system.

Therefore, for the first case, the best solution for $\mathrm{k}=1$, are the three optimal dimensions $t_{p}=0.8710, t_{h}=0.8761$, and $t_{\nu}=1.3103$. While for $\mathrm{k}=2$, the three optimal dimensions are $t_{p}=0.9963, t_{h}=0.8334$, and $t_{\nu}=1.2042$. These results validate those found analytically by [14]. For the second case, it was found that after $\mathrm{k}=$ 16 operations, the optimal dimensions are practically constant, i.e. $t_{h} €[0.4585$ $0.4699], t_{p} €[1.2058-1.2177]$ and $t_{v} €[1.7559-1.7909]$. Moreover, the least squares method was applied to find functions that relate the optimal values $\left(t_{p}, t_{h}\right.$, and $t_{v}$ ) to the number of operations $\mathrm{k}$, in order to obtain expressions allowing a direct evaluation of the three optimal dimensions of the system.

Furthermore, the proposed solutions allow greater flexibility when choosing dimensions for the design of a multi-aisle AS/RS with a multi-shuttle S/R machine.

Regarding the possible future perspectives of research in the field, it would be desirable to compare these results, found by the genetic algorithm, with others determined through different metaheuristics, in the case of a multi cycle time. It would also be interesting to find the optimal dimensions of another type of storage and retrieval system.

\section{A Appendix}

We present the 18 equations of the time between two bins in the multi-aisle AS/RS. In the case where the storage and retrieval are done on different aisles as following:

$$
\begin{gathered}
\frac{1}{1260} \frac{420 t_{h}^{2} t_{p}^{3}+1260 t_{h}^{3} t_{p}^{2}+7 t_{v}^{4} t_{p}-t_{v}^{5}}{t_{h}^{2} t_{p}^{2}} t_{p} \leq t_{h} \text { and } 0<t_{v} \text { and } t_{v} \leq t_{p} \\
\frac{1}{1260} \frac{\left(21 t_{v}^{5}-35 t_{v}^{4} t_{p}+35 t_{p}^{2} t_{v}^{3}-21 t_{v}^{2} t_{p}^{3}+1260 t_{v}^{2} t_{h}^{3}+7 t_{v} t_{p}^{4}+420 t_{h}^{3} t_{p} t_{v}^{2}-t_{p}^{5}\right)}{t_{h}^{2} t_{p}^{2}} \\
t_{p} \leq t_{h} \text { and } t_{p}<t_{v} \text { and } t_{v} \leq t_{h}
\end{gathered}
$$




$$
\begin{aligned}
& \frac{-1}{1260} \frac{1}{t_{h}^{2} t_{p}^{2} t_{v}^{2}}\left(t_{p}^{7}-84 t_{h}^{5}\left(t_{p} t_{v}-7 t_{p}^{6} t_{v}-84 t_{h} t_{p} t_{\nu}^{5}+14 t_{h} t_{v}^{6}+14 t_{p} t_{v}^{6}\right.\right. \\
& +14 t_{h}^{6} t_{p}-14 t_{h}^{6} t_{v}-21 t_{p}^{2} t_{v}^{5}-42 t_{h}^{2} t_{v}^{5}+70 t_{h}^{3} t_{v}^{4}+35 t_{p}^{3} t_{v}^{4}-70 t_{h}^{4} t_{v}^{3} \\
& -35 t_{p}^{4} t_{v}^{3}+42 t_{v}^{2} t_{h}^{5}+21 t_{v}^{2} t_{p}^{5}+210 t_{h}^{2} t_{p} t_{v}^{4}-280 t_{h}^{3} t_{p} t_{v}^{3}+210 t_{\nu}^{2} t_{p} t_{h}^{4} \\
& \left.-1260 t_{h}^{3} t_{v}^{2} t_{p}^{2}-420 t_{h}^{2} t_{v}^{2} t_{p}^{3}-2 t_{v}^{7}+2 t_{h}^{7}\right) \\
& t_{p} \leq t_{h} \text { and } t_{h}<t_{v} \text { and } t_{v} \leq t_{h}+2 t_{h}
\end{aligned}
$$

$$
\begin{aligned}
& \frac{-1}{1260} \frac{1}{t_{h}^{2} t_{v}^{2}}\left(v^{2} t_{p}^{3}-21 t_{v}^{5}-420 t_{h}^{2} t_{v}^{3}-35 t_{p}^{2} t_{v}^{3}-7 t_{v} t_{p}^{4}+35 t_{v}^{4} t_{p}+210 t_{h} t_{v}^{4}\right. \\
& -210 t_{h}^{4} t_{v}-280 t_{p} t_{h}^{3} t_{v}-210 t_{v} t_{h}^{2} t_{p}^{2}-84 t_{v} t_{h} t_{p}^{3}+840 t_{h}^{2} t_{p} t_{v}^{2}-280 t_{h} t_{p} \\
& \left.t_{v}^{3}+14 t_{h} t_{p}^{4}+42 t_{h}^{5}+70 t_{p} t_{h}^{4}+70 t_{h}^{3} t_{p}^{2}+42 t_{h}^{2} t_{p}^{3}+1680 t_{v}^{2} t_{h}^{3}+210 t_{v}^{2} t_{h} t_{p}^{2}+t_{p}^{5}\right) \\
& t_{p} \leq t_{h} \text { and } t_{p}+t_{h}<t_{v} \text { and } t_{v} \leq 2 t_{h} \\
& \frac{1}{90} \frac{\left(-30 t_{v}^{3}-60 t_{h} t_{p} t_{v}-105 t_{v} t_{h}^{2}-15 t_{p}^{2} t_{v}+45 t_{h}^{3}+35 t_{h}^{2} t_{p}+15 t_{h} t_{p}^{2}+3 t_{p}^{3}\right)}{t_{v}^{2}}
\end{aligned}
$$

$t_{p} \leq t_{h}$ and $t_{p}+2 t_{h}<t_{\nu}$

$$
\begin{aligned}
& \frac{-1}{1260} \frac{1}{t_{h}^{2} t_{p}^{2}}\left(336 t_{h}^{5}-1680 t_{p} t_{h}^{4}+2520 t_{h}^{3} t_{p}^{2}-840 t_{h}^{2} t_{p}^{3}-1050 t_{h} t_{p}^{4}\right. \\
& \left.+2520 t_{h} t_{p}^{3}+840 t_{p} t_{h}^{3}+t_{\nu}^{5}+252 t_{p}^{5}-420 t_{p}^{4}-7 t_{\nu}^{4} t_{p}-3780 t_{h}^{2} t_{p}^{2}\right) \\
& t_{h}<t_{p} \text { and } t_{p} \leq 2 t_{h} \text { and } 0<t_{p} \text { and } t_{\nu} \leq t_{h}
\end{aligned}
$$

$$
\begin{aligned}
& \frac{1}{1260} \frac{1}{t_{h}^{2} t_{p}^{2} t_{v}^{2}}\left(-84 t_{h}^{5} t_{p} t_{v}+70 t_{h}^{3} t_{v}^{4}-42 t_{h}^{2} t_{v}^{5}-14 t_{h}^{6} t_{v}+14 t_{h}^{6} t_{p}-t_{v}^{7}-84 t_{h}\right. \\
& t_{p} t_{v}^{5}+210 t_{h}^{2} t_{p} t_{v}^{4}-280 t_{h}^{3} t_{p} t_{v}^{3}+42 t_{v}^{2} t_{h}^{5}+2 t_{h}^{7}+14 t_{h} t_{v}^{6}+7 t_{p} t_{v}^{6}-70 t_{h}^{4} t_{v}^{3} \\
& \left.+210 t_{v}^{2} t_{p} t_{h}^{4}-1260 t_{h}^{3} t_{v}^{2} t_{p}^{2}-420 t_{h}^{2} t_{v}^{2} t_{p}^{3}\right) \\
& t_{h}<t_{p} \text { and } t_{p} \leq 2 t_{h} \text { and } t_{h}<t_{v} \text { and } t_{v} \leq t_{p} \\
& \quad \frac{1}{1260} \frac{1}{t_{h}^{2} t_{p}^{2} t_{v}^{2}}\left(t_{p}^{7}-84 t_{h}^{5} t_{p} t_{v}-7 t_{p}^{6} t_{v}-84 t_{h} t_{p} t_{h}^{5}+14 t_{h} t_{h}^{6}+14 t_{p} t_{h}^{6}\right. \\
& \quad+14 t_{h}^{6} t_{p}-14 t_{h}^{6} t_{v}-21 t_{p}^{2} t_{h}^{4}-42 t_{h}^{2} t_{h}^{5}+70 t_{h}^{3} t_{h}^{4}+35 t_{p}^{3} t_{h}^{4}-70 t_{h}^{4} t_{h}^{3} \\
& \quad-35 t_{p}^{4} t_{h}^{3}+42 t_{h}^{2} t_{h}^{5}+21 t_{h}^{2} t_{p}^{5}+210 t_{h}^{2} t_{p} t_{h}^{4}-280 t_{h}^{3} t_{p} t_{h}^{3}+210 t_{h}^{2} t_{p} t_{h}^{4} \\
& \left.\quad-1260 t_{h}^{3} t_{h}^{2} t_{p}^{2}-420 t_{h}^{2} t_{h}^{2} t_{p}^{3}-2 t_{h}^{7}+2 t_{h}^{7}\right) \\
& t_{h}<t_{p} \text { and } t_{p} \leq 2 t_{h} \text { and } t_{p}<t_{v} \text { and } t_{v} \leq 2 t_{h}
\end{aligned}
$$




$$
\begin{aligned}
& \frac{1}{1260} \frac{1}{t_{h}^{2} t_{p}^{2} t_{v}^{2}}\left(630 t_{v}^{2} t_{h}^{5}+35 t_{p}^{4} t_{v}^{3}+1260 t_{h}^{3} t_{v}^{2} t_{p}^{2}-t_{p}^{7}+t_{v}^{7}-1260 t_{h}^{5} t_{p} t_{v}-7 t_{p} t_{v}^{6}\right. \\
& +126 t_{h}^{7}+420 t_{h}^{2} t_{v}^{2} t_{p}^{3}+210 t_{h}^{2} t_{p} t_{v}^{4}-840 t_{h}^{3} t_{p} t_{v}^{3}+1470 t_{v}^{2} t_{p} t_{h}^{4}-434 t_{h}^{6} t_{v}-35 t_{p}^{3} t_{v}^{4} \\
& \left.+210 t_{h}^{3} t_{v}^{4}-490 t_{h}^{4} t_{v}^{3}+21 t_{p}^{2} t_{v}^{5}+7 t_{p}^{6} t_{v}-21 t_{v}^{2} t_{p}^{5}-42 t_{h}^{2} t_{v}^{5}+434 t_{h}^{6} t_{p}\right) \\
& t_{h}<t_{p} \text { and } t_{p} \leq 2 t_{h} \text { and } 2 t_{h}<t_{v} \text { and } t_{v} \leq t_{p}+t_{h}
\end{aligned}
$$

$$
\begin{aligned}
& \frac{1}{1260} \frac{1}{t_{h}^{2} t_{p}^{2} t_{v}^{2}}\left(448 t_{h}^{2} t_{p}-448 t_{h}^{6} t_{v}-21 t_{p}^{2} t_{v}^{5}+42 t_{h}^{2} t_{p}^{5}+7 t_{p} t_{v}^{6}-84 t_{h}^{2} t_{v}^{5}+280 t_{h}^{3} t_{v}^{4}+35 t_{p}^{3} t_{v}^{4}\right. \\
& -560 t_{h}^{4} t_{v}^{3}-35 t_{p}^{4} t_{v}^{3}+672 t_{v}^{2} t_{h}^{5}+21 t_{v}^{2} t_{p}^{5}+14 t_{h} t_{p}^{6}+70 t_{h}^{3} t_{p}^{4}+70 t_{p}^{3} t_{h}^{4}+42 t_{p}^{2} t_{h}^{5}+1680 t_{h}^{3} t_{v}^{2} t_{p}^{2} \\
& +840 t_{h}^{2} t_{v}^{2} t_{p}^{3}-84 t_{h} t_{p}^{5} t_{v}-280 t_{h} t_{p}^{3} t_{v}^{3}+210 t_{h} t_{p}^{2} t_{v}^{4}-210 t_{h}^{4} t_{v} t_{p}^{2}-210 t_{p}^{4} t_{v} t_{h}^{2}-420 t_{h}^{2} t_{p}^{2} t_{v}^{3} \\
& -280 t_{h}^{3} t_{p}^{3} t_{v}+210 t_{\nu}^{2} t_{h} t_{p}^{4}+1680 t_{v}^{2} t_{p} t_{h}^{4}-1120 t_{h}^{3} t_{p} t_{v}^{3}+420 t_{h}^{2} t_{p} t_{v}^{4} \\
& \left.-84 t_{h} t_{p} t_{v}^{5}-1344 t_{h}^{5} t_{p} t_{v}+t_{p}^{7}-t_{v}^{7}+128 t_{h}^{7}-7 t_{p}^{6} t_{v}+14 t_{h} t_{v}^{6}\right) \\
& t_{h}<t_{p} \text { and } t_{p} \leq 2 t_{h} \text { and } t_{p}+t_{h}<t_{\nu} \text { and } t_{v} \leq t_{p}+2 t_{h}
\end{aligned}
$$$$
\frac{-1}{90} \frac{\left(-30 t_{v}^{3}-60 t_{h} t_{p} t_{v}-105 t_{v} t_{h}^{2}-15 t_{v}^{2} t_{v}+45 t_{h}^{3}+35 t_{h}^{2} t_{p}+15 t_{h} t_{v}^{2}+3 t_{v}^{3}\right)}{t_{v}^{2}}
$$

$$
\begin{aligned}
& t_{h}<t_{p} \text { and } t_{p} \leq+2 t_{h} \text { and } t_{p}+2 t_{h}<t_{v} \\
& \frac{-1}{90} \frac{\left(420 t_{h}^{2} t_{p}^{3}+1260 t_{h}^{3} t_{p}^{2}+7 t_{v}^{4} t_{p}-t_{v}^{5}\right)}{t_{h}^{2}+t_{p}^{2}} \\
& 2 t_{h}<t_{p} \text { and } 0<t_{v} \text { and } t_{v} \leq t_{h} \\
& \frac{-1}{1260} \frac{1}{t_{h}^{2} t_{p}^{2} t_{v}^{2}}\left(-84 t_{h}^{5} t_{p} t_{v}+70 t_{h}^{3} t_{v}^{4}-42 t_{h}^{2} t_{v}^{5}-14 t_{h}^{6} t_{v}+14 t_{h}^{6} t_{p}-t_{v}^{7}\right. \\
& -84 t_{h} t_{p} t_{v}^{5}+210 t_{h}^{2} t_{p} t_{v}^{4}-280 t_{h}^{3} t_{p} t_{v}^{3}+42 t_{v}^{2} t_{h}^{5}+2 t_{h}^{7}+14 t_{h} t_{v}^{6}+7 t_{p} t_{v}^{6} \\
& \left.-70 t_{h}^{4} t_{v}^{3}+210 t_{v}^{2} t_{p} t_{h}^{4}-1260 t_{h}^{3} t_{v}^{2} t_{p}^{2}-420 t_{h}^{2} t_{v}^{2} t_{p}^{3}\right) \\
& 2 t_{h}<t_{p} \text { and } t_{h}<t_{p} \text { and } t_{v}<2 t_{h} \\
& \frac{1}{90} \frac{1}{t_{p}^{2} t_{v}^{2}}\left(90 t_{\nu}^{2} t_{h} t_{p}^{2}-60 t_{h} t_{p} t_{v}^{3}+9 t_{h}^{5}+15 t_{v}^{4} t_{p}+45 t_{v}^{2} t_{h}^{3}\right. \\
& -31 t_{h}^{4} t_{v}+31 t_{p} t_{h}^{4}-3 t_{v}^{5}+15 t_{h} t_{v}^{4}-35 t_{h}^{2} t_{v}^{3}+105 t_{h}^{2} t_{p} t_{v}^{2} \\
& \left.+30 t_{v}^{2} t_{p}^{3}-90 t_{p} t_{h}^{3} t_{v}\right) \\
& 2 t_{h}<t_{p} \text { and } 2 t_{h}<t_{v} \text { and } t_{v} \leq t_{p}
\end{aligned}
$$




$$
\begin{aligned}
& \frac{1}{1260} \frac{1}{t_{h}^{2} t_{p}^{2} t_{v}^{2}}\left(630 t_{v}^{2} t_{h}^{5}+35 t_{p}^{4} t_{v}^{3}+1260 t_{h}^{3} t_{v}^{2} t_{p}^{2}-t_{p}^{7}+t_{v}^{7}-1260 t_{h}^{5} t_{p} t_{v}\right. \\
& -7 t_{p} t_{v}^{6}+126 t_{h}^{7}+420 t_{h}^{2} t_{v}^{2} t_{p}^{3}+210 t_{h}^{2} t_{p} t_{v}^{4}-840 t_{h}^{3} t_{p} t_{v}^{3}+1470 t_{v}^{2} t_{p} t_{h}^{4} \\
& -434 t_{h}^{6} t_{v}-35 t_{p}^{3} t_{v}^{4}+210 t_{h}^{3} t_{v}^{4}-490 t_{h}^{4} t_{v}^{3}+21 t_{p}^{2} t_{v}^{5}+7 t_{p}^{6} t_{v}-21 t_{v}^{2} t_{p}^{5} \\
& \left.-42 t_{h}^{2} t_{v}^{5}+434 t_{h}^{6} t_{p}\right) \\
& 2 t_{h}<t_{p} \text { and } t_{p}<t_{v} \text { and } t_{v} \leq t_{p}+t_{h} \\
& \frac{1}{1260} \frac{1}{t_{h}^{2} t_{p}^{2} t_{v}^{2}}\left(448 t_{h}^{6} t_{p}-448 t_{h}^{6} t_{v}-21 t_{p}^{2} t_{v}^{5}+42 t_{h}^{2} t_{p}^{5}+7 t_{p} t_{v}^{6}-84 t_{h}^{2} t_{v}^{5}\right. \\
& +280 t_{h}^{3} t_{v}^{4}+35 t_{p}^{3} t_{v}^{4}-560 t_{h}^{4} t_{v}^{3}-35 t_{p}^{4} t_{v}^{3}+672 t_{v}^{2} t_{h}^{5}+21 t_{v}^{2} t_{p}^{5}+14 t_{h} t_{p}^{6} \\
& +70 t_{h}^{3} t_{p}^{4}+70 t_{p}^{3} t_{h}^{4}+42 t_{p}^{2} t_{h}^{5}+1680 t_{h}^{3} t_{v}^{2} t_{p}^{2}+840 t_{h}^{2} t_{v}^{2} t_{p}^{3}-84 t_{h} t_{p}^{5} t_{v} \\
& -280 t_{h} t_{p}^{3} t_{v}^{3}+210 t_{h} t_{p}^{2} t_{v}^{4}-210 t_{h}^{4} t_{v} t_{p}^{2}-210 t_{p}^{4} t_{v} t_{h}^{2}-420 t_{h}^{2} t_{p}^{2} t_{v}^{3} \\
& -280 t_{h}^{3} t_{p}^{3} t_{v}+210 t_{v}^{2} t_{h} t_{p}^{4}+1680 t_{v}^{2} t_{p} t_{h}^{4}-1120 t_{h}^{3} t_{p} t_{v}^{3}+420 t_{h}^{2} t_{p} t_{v}^{4} \\
& \left.-84 t_{h} t_{p} t_{v}^{5}-1344 t_{h}^{5} t_{p} t_{v}+t_{p}^{7}-t_{v}^{7}+128 t_{h}^{7}-7 t_{p}^{6} t_{v}+14 t_{h} t_{v}^{6}\right) \\
& 2 t_{h}<t_{p} \text { and } t_{p}+t_{h}<t_{v} \text { and } t_{v} \leq t_{p}+2 t_{h} \\
& \frac{-1}{90} \frac{\left(-30 t_{\nu}^{3}-60 t_{h} t_{p} t_{v}-105 t_{v} t_{h}^{2}-15 t_{p}^{2} t_{v}+45 t_{h}^{3}+35 t_{h}^{2} t_{p}+15 t_{h} t_{p}^{2}+3 t_{p}^{3}\right)}{t_{v}^{2}}
\end{aligned}
$$

$$
2 t_{h}<t_{p} \text { and } t_{p}+2 t_{h}<t_{v}
$$

\section{B Declarations}

a. Funding: No funding was received to assist with the preparation of this manuscript .

b. Conflicts of interest/Competing interests: The authors have no conflicts of interest to declare that are relevant to the content of this article.

c. Availability of data and material: Not applicable for that section.

d. Code availability: Not applicable for that section.

e. Authors' contributions: I confirm that all authors listed on the title page have contributed significantly to the work, have read the manuscript, attest to the validity and legitimacy of the data and its interpretation, and agree to its submission.

f. Ethics approval: Not applicable for that section.

g. Consent to participate: Not applicable for that section.

h. Consent for publication: Not applicable for that section. 


\section{References}

1. Azzi, A., Battini, D., Faccio, M., Persona, A., Sgarbossa, F.: Innovative travel time model for dualshuttle automated storage/retrieval systems. Computers \& Industrial Engineering 61 (3), 600-607 (2011)

2. Bessenouci, H.N., Sari, Z., Ghomri, L.: Metaheuristic based control of a flow rack automated storage retrieval system. Journal of Intelligent Manufacturing 23(4), 1157-1166 (2012)

3. Borovinšek, M., Ekren, B.Y., Burinskienè, A., Lerher, T.: Multi-objective optimisation model of shuttle-based storage and retrieval system. Transport 32(2), 120-137 (2017)

4. Bozer, Y.A., White, J.A.: Travel-time models for automated storage/retrieval systems. IIE transactions 16(4), 329-338 (1984)

5. Brezovnik, S., Gotlih, J., Balič, J., Gotlih, K., Brezočnik, M.: Optimization of an automated storage and retrieval systems by swarm intelligence. Procedia Engineering 100, 1309-1318 (2015)

6. Cunkas, M., Ozer, O.: Optimization of location assignment for unit-load as/rs with a dual-shuttle. International Journal of Intelligent Systems and Applications in Engineering 7(2), 66-71 (2019)

7. Dooly, D.R., Lee, H.F: A shift-based sequencing method for twin-shuttle automated storage and retrieval systems. IIE Transactions 40(6), 586-594 (2008)

8. Dube, C.: Calculating the cost of asrs: 5 contributing factors. (accessed 07 jully 2021) (2021). URL https://us.blog.kardex-remstar.com/asrs-cost-factors

9. Ghomri, L., Sari, Z., Guezzen, A.H., Sari, T.: Expressions analytiques du temps de cycle pour les as/rs multi allées. In: Actes de La 7e Conférence Internationale de Modélisation et Simulation, vol. 1, pp. 333-340 (2008)

10. Hamzaoui, M.A., Sari, Z.: Optimal dimensions minimizing expected travel time of a single machine flow rack as/rs. Mechatronics 31, 158-168 (2015)

11. Hamzaoui, M.A., Zaki, S.: Cycle time models for the bidirectional flow-rack as/rs. FME Transactions 48(1), 211-226 (2020)

12. Holland, J.: Evolution in natural and artificial systems. Ann Arbor: U. of Michigan Press (1975)

13. Hwang, H., KO, C.S.: A study on multi-aisle system served by a single storage/retrieval machine. The International Journal Of Production Research 26(11), 1727-1737 (1988)

14. Kouloughli, S., Sari, Z.: Multi-aisle as/rs dimensions optimization for cycle time minimization. The International Journal of Advanced Manufacturing Technology 79(1-4), 675-692 (2015)

15. Kouloughli, S., Sari, Z., Sari, T.: Optimisation des dimensions d'un as/rs multi-allée basée sur un modèle analytique du temps de simple cycle. Journal Européen des Systèmes Automatisés (JESA) 44(2), 135-159 (2010)

16. Lerher, T., Sraml, M., Kramberger, J., Potrc, I., Borovinsek, M., Zmazek, B.: Analytical travel time models for multi aisle automated storage and retrieval systems. The international journal of advanced manufacturing technology 30(3-4), 340-356 (2006)

17. Meller, R.D., Mungwattana, A.: Multi-shuttle automated storage/retrieval systems. IIE transactions 29(10), 925-938 (1997)

18. Metahri, D., Hachemi, K.: Retrieval-travel-time model for free-fall-flow-rack automated storage and retrieval system. Journal of Industrial Engineering International 14(4), 807-820 (2018)

19. Mostofi, A., Erfanian, H.: Multi-shuttle automated storage and retrieval system (2018)

20. Popović, D., Vidović, M., Bjelić, N.: Application of genetic algorithms for sequencing of as/rs with a triple-shuttle module in class-based storage. Flexible services and manufacturing journal 26(3), 432-453 (2014)

21. Potrč, I., Lerher, T., Kramberger, J., Šraml, M.: Simulation model of multi-shuttle automated storage and retrieval systems. Journal of Materials Processing Technology 157, 236-244 (2004)

22. Schenone, M., Mangano, G., Grimaldi, S., Cagliano, A.C.: An approach for computing as/r systems travel times in a class-based storage configuration. Production \& Manufacturing Research 8(1), 273-290 (2020)

23. Xu, X., Gong, Y., Fan, X., Shen, G., Zou, B.: Travel-time model of dual-command cycles in a 3d compact as/rs with lower mid-point i/o dwell point policy. International Journal of Production Research 56(4), 1620-1641 (2018)

24. Yang, P., Miao, L., Xue, Z., Qin, L.: An integrated optimization of location assignment and storage/retrieval scheduling in multi-shuttle automated storage/retrieval systems. Journal of Intelligent Manufacturing 26(6), 1145-1159 (2015) 